\title{
Quantum characteristic classes and the Hofer metric
}

\author{
YASHA SAVELYEV
}

\begin{abstract}
Given a closed monotone symplectic manifold $M$, we define certain characteristic cohomology classes of the free loop space $\operatorname{LHam}(M, \omega)$ with values in $Q H_{*}(M)$, and their $S^{1}$ equivariant version. These classes generalize the Seidel representation and satisfy versions of the axioms for Chern classes. In particular there is a Whitney sum formula, which gives rise to a graded ring homomorphism from the ring $H_{*}(\Omega \operatorname{Ham}(M, \omega), \mathbb{Q})$, with its Pontryagin product to $Q H_{2 n+*}(M)$ with its quantum product. As an application we prove an extension to higher dimensional geometry of the loop space $L \operatorname{Ham}(M, \omega)$ of a theorem of McDuff and Slimowitz on minimality in the Hofer metric of a semifree Hamiltonian circle action.
\end{abstract}

53D45; 53D35, 22E67

\section{Introduction}

The topology and geometry of the group $\operatorname{Ham}(M, \omega)$ of Hamiltonian symplectomorphisms of a symplectic manifold $M$ has been intensely studied by numerous authors. This is an infinite-dimensional manifold with a remarkable bi-invariant Finsler metric induced by the Hofer norm. It is of foundational importance in symplectic geometry and Hamiltonian mechanics. As of now the deepest insights into the topology and Hofer geometry of $\operatorname{Ham}(M, \omega)$ come from Gromov-Witten invariants and related quantum and Floer homology constructions. Still, rather little general information is known.

We define some general invariants, which will be used to $\operatorname{study} \operatorname{Ham}(M, \omega)$. The Hofer geometry will serve a unifying role, and ultimately is what allows us to compute the invariants in some cases. These computations can in turn be used to get Hofer geometric and topological information. What follows is a rapid review of Hofer geometry.

\subsection{Hofer geometry and Seidel representation}

Given a smooth function $H_{t}: M \rightarrow \mathbb{R}, 0 \leq t \leq 1$, there is an associated time dependent Hamiltonian vector field $X_{t}, 0 \leq t \leq 1$, defined by

$$
\omega\left(X_{t}, \cdot\right)=d H_{t}(\cdot) \text {. }
$$


The vector field $X_{t}$ generates a path $\gamma_{t}, 0 \leq t \leq 1$, in $\operatorname{Diff}(M, \omega)$. Given such a path $\gamma_{t}$, its endpoint $\gamma_{1}$ is called a Hamiltonian symplectomorphism. The space of Hamiltonian symplectomorphisms forms a group, denoted by $\operatorname{Ham}(M, \omega)$.

In particular the path $\gamma_{t}$ above lies in $\operatorname{Ham}(M, \omega)$. It is well known that any path $\left\{\gamma_{t}\right\}$ in $\operatorname{Ham}(M, \omega)$ with $\gamma_{0}=\mathrm{id}$ arises in this way (is generated by $H_{t}: M \rightarrow \mathbb{R}$ ). Given such a path $\left\{\gamma_{t}\right\}$, the Hofer length $L\left(\gamma_{t}\right)$ is defined by

$$
L\left(\gamma_{t}\right):=\int_{0}^{1} \max \left(H_{t}^{\gamma}\right)-\min \left(H_{t}^{\gamma}\right) d t
$$

where $H_{t}^{\gamma}$ is a generating function for the path $\gamma_{0}^{-1} \gamma_{t}, 0 \leq t \leq 1$. The Hofer distance $\rho(\phi, \psi)$ is defined by taking the infimum of the Hofer length of paths from $\phi$ to $\psi$. It is a deep theorem that the resulting metric is nondegenerate (see Hofer [5] and Lolonde and $\operatorname{McDuff}[10])$. This gives $\operatorname{Ham}(M, \omega)$ the structure of a Finsler manifold. We will be more concerned with a related measure of the path

$$
L^{+}\left(\gamma_{t}\right):=\int_{0}^{1} \max \left(H_{t}^{\gamma}\right),
$$

where $H_{t}^{\gamma}$ is in addition normalized by the condition

$$
\int_{M} H^{\gamma_{t}}=0
$$

Let $\gamma: S^{1} \rightarrow \operatorname{Ham}(M, \omega)$ be a subgroup with generating Hamiltonian $H$. Let $F_{\max }, F_{\min }$ denote the maximum and minimum level sets of $H$ (these are fixed by $\gamma)$. We say that $\gamma$ is semifree at $F_{\max }$, respectively $F_{\min }$, if all the nonzero weights of the linearized action of $\gamma$ on the normal bundles to $F_{\max }$, respectively $F_{\min }$, are -1 , respectively +1 . To define these weights we take an $S^{1}$ equivariant orientation preserving identification of the normal bundle to $F_{\max }$ at $x \in F_{\max }$ with $\mathbb{C}^{m}$, for some $m$, which splits into $\gamma$ invariant 1-complex-dimensional subspaces $N_{k_{i}}$, on which $\gamma$ is acting by

$$
v \mapsto e^{-2 \pi i k_{i} \theta} v
$$

These $k_{i}$ are then defined to the weights of the circle action $\gamma$. Here is one theorem that does give some general information about topology and geometry of $\operatorname{Ham}(M, \omega)$.

Theorem 1.1 (McDuff-Slimowitz-Tolman $[18 ; 19]$ ) Let $\gamma$ be a Hamiltonian circle action on a symplectic manifold $M$, which is semifree at $F_{\max }$ and $F_{\min }$. Then $\gamma$ is length-minimizing in its homotopy class for the Hofer metric on $\operatorname{Ham}(M, \omega)$. 
One of the motivating applications of this thesis is an extension of this theorem to the higher-dimensional geometry of $\operatorname{Ham}(M, \omega)$. The starting point for this is the Seidel representation defined in [24]. This is a homomorphism

$$
S: \pi_{1}(\operatorname{Ham}(M, \omega)) \rightarrow Q H_{2 n}^{\times}(M),
$$

where $Q H_{2 n}^{\times}(M)$ denotes the group of multiplicative units of degree $2 \mathrm{n}$ in quantum homology $Q H_{*}(M)$, and $2 n$ is the dimension of $M$. This representation of $\pi_{1}(\operatorname{Ham}(M, \omega))$ is a powerful tool in understanding the symplectic geometry of the manifold $(M, \omega)$, of Hamiltonian fibrations $X_{\phi}$ over $S^{2}$ associated to loops $\left\{\phi_{t}\right\}$ in $\operatorname{Ham}(M, \omega)$, as well as the Hofer geometry and topology of the group $\operatorname{Ham}(M, \omega)$. In particular, Theorem 1.1 can be proved using the Seidel representation as is essentially done by McDuff and Tolman [19].

There is a completely natural extension of Seidel representation to certain cohomology classes of the associated loop spaces of $\operatorname{Ham}(M, \omega)$.

\subsection{Quantum characteristic classes}

Consider the free loop space $L \operatorname{Ham}(M, \omega)$, which we will abbreviate by $L \mathrm{Ham}$. We construct natural bundles

$$
\tilde{p}: U \rightarrow L \text { Ham } \quad \text { and } \quad p: U^{S^{1}} \rightarrow Q \equiv\left(L \operatorname{Ham} \times S^{\infty}\right) / S^{1} .
$$

The fiber over a loop $\gamma$ is modelled by a Hamiltonian fibration $\pi: X_{\gamma} \rightarrow S^{2}$, with fiber $M$, associated to the loop $\gamma$ as follows:

$$
X_{\gamma}=\left(M \times D_{0}^{2}\right) \cup\left(M \times D_{\infty}^{2}\right) / \sim
$$

where the equivalence relation $\sim$ is: $(x, 1, \theta)_{0} \sim\left(\gamma_{\theta}(x), 1, \theta\right)_{\infty}$. Here $D_{0}^{2}$ and $D_{\infty}^{2}$ are two names for the unit disk $D^{2} \subset \mathbb{C}$ and $(r, 2 \pi \theta)$ are polar coordinates on $D$.

Let $p: P \rightarrow B$ be a bundle obtained by pullback of either $\tilde{p}: U \rightarrow L$ Ham or $p: U^{S^{1}} \rightarrow$ $Q$, where $B$ is a closed oriented smooth manifold. The bundle $P$ comes with a natural deformation class of families of symplectic forms $\left\{\Omega_{b}\right\}$ on the fibers $\left\{X_{b}\right\}$. We will define characteristic classes

$$
c_{k}^{q}(P) \in H^{k}\left(B, Q H_{*}(M)\right),
$$

by counting the number of fiber-wise or vertical $J$-holomorphic curves passing through certain natural homology classes in $P$. Here $k$ is the degree of the class and the superscript $q$ stands for quantum to distinguish it from the Chern classes $c_{k}$. 
Remark 1.2 Recall that the top Chern class of a complex vector bundle is its Euler class, whose Poincare dual is represented by the self intersection of the zero section. The classes $c_{k}^{q}(P)$ are also in a sense described by the self intersection of a natural homology class in $P$, playing the role of the zero section, except that the classical intersection is always empty and instead one keeps track of "instanton (or quantum) corrections" to this self-intersection, coming from the presence of vertical $J$-holomorphic spheres. This is the motivation for the name quantum characteristic class (cf Vafa [26]).

Remark 1.3 Michael Hutchings discovered maps

$$
\pi_{k}(\operatorname{Ham}(M, \omega)) \rightarrow \operatorname{End}_{k-1}\left(Q H_{*}(M)\right),
$$

where $\operatorname{End}_{k-1}\left(Q H_{*}(M)\right)$ denotes the additive group of endomorphisms of the quantum homology of degree $k-1$. These maps generalize the Seidel representation. In fact his project is much more extensive [6;7]. Hutchings defines (4) as a kind of a "family" or "higher" continuation map in Floer homology. Dusa McDuff [13] suggested the following approach to these maps. Glue together $M \times\left(\mathbb{C P}^{k} \backslash B\right)$ with $M \times \bar{B}$, where $B$ is an open $2 k$ dimensional ball, by using a map $f: S^{2 k-1} \rightarrow \operatorname{Ham}(M, \omega)$ as a clutching map. We get a certain Hamiltonian fibration with fiber $M$ over $\mathbb{C P}^{k}$ and may presumably define a parametrized variant of the Seidel representation which takes into account the natural family of holomorphic curves in $\mathbb{C} \mathbb{P}^{k}$. We are motivated by this idea but our approach is slightly more abstract, which also leads to a close relationship with the Hofer metric. We expect that our invariants will coincide with Hutchings' for cycles in the loop space $\Omega \operatorname{Ham}(M, \omega)$ induced by homotopy classes in $\pi_{k}(\operatorname{Ham}(M, \omega))(\mathrm{cf}$ Example 2.8 and also Theorem 1.15).

Definition 1.4 Given fibrations $P_{f_{1}}, P_{f_{2}}$ over $B$, induced by maps

$$
f_{1}, f_{2}: B \rightarrow L \operatorname{Ham}(M, \omega)
$$

we define their sum $P_{f_{1}} \oplus P_{f_{2}}$ to be $P_{f_{2} \cdot f_{1}}$, where $f_{2} \cdot f_{1}: B \rightarrow L \operatorname{Ham}(M, \omega)$ is the pointwise product of the maps $f_{1}, f_{2}$ induced by the topological group structure of $L \operatorname{Ham}(M, \omega)$.

We'll show in Section 7 that these fibrations have a natural structure group $\mathcal{F}$ and that $\operatorname{LHam}(M, \omega)$ is the classifying space of this structure group. Let $\mathcal{P}_{\boldsymbol{B}, M}$ denote the group of isomorphism classes of fibrations $p: P \rightarrow B$ with structure group $\mathcal{F}$ (ie the group of homotopy classes of maps $f: B \rightarrow L$ Ham).

We may now state the axioms satisfied by our characteristic classes. For simplicity we assume here that the base $B$ is connected. 
Definition 1.5 Quantum characteristic classes are a sequence of functions

$$
c_{k}^{q}: \mathcal{P}_{B, M} \rightarrow H^{k}\left(B, Q H_{*}(M)\right),
$$

satisfying the following axioms:

Axiom 1 (Partial normalization) $c_{0}^{q}(P)=S([\gamma])$ if the fiber of $p: P \rightarrow B$ is modelled on $X_{\gamma}$, where $S$ is the Seidel representation. Further if $P$ is trivial then $c_{k}^{q}(P)=0$ for $k>0$.

Axiom 2 (Functoriality) If $g: B_{1} \rightarrow B_{2}$ is smooth, then

$$
g^{*}\left(c_{k}^{q}\left(P_{2}\right)\right)=c_{k}^{q}\left(g^{*}\left(P_{2}\right)\right) .
$$

Axiom 3 (Whitney sum formula) If $P=P_{1} \oplus P_{2}$, then

$$
c^{q}(P)=c^{q}\left(P_{1}\right) \cup c^{q}\left(P_{2}\right),
$$

where $U$ is the cup product of cohomology classes with coefficients in the quantum homology ring $Q H_{*}(M)$ and $c^{q}(P)$ is the total characteristic class

$$
c^{q}(P)=c_{0}^{q}(P)+\ldots+c_{m}^{q}(P),
$$

where $m$ is the dimension of $B$. (In practice, we mainly deal with the identity component of LHam. In this case $c_{0}^{q}(P)=S([\gamma])$ is the identity $[M]$ in the quantum homology ring and so we get an expression in (5) analogous to the total Chern class.)

Theorem 1.6 If $(M, \omega)$ is a closed monotone symplectic manifold, then there exist natural nontrivial quantum characteristic classes

$$
c_{k}^{q}: \mathcal{P}_{B, M} \rightarrow H^{k}\left(B, Q H_{*}(M)\right) .
$$

We define these classes in Section 3 and prove in Section 4 there that they satisfy Axioms 1,2 and 3.

Remark 1.7 Throughout we work with the class of monotone symplectic manifolds $(M, \omega)$, ie those satisfying

$$
[\omega]=\text { const } \cdot c_{1}(M),
$$

for a const $>0$. This condition insures that the relevant evaluation maps are pseudocycles. It is likely that this condition can be removed in the definition of QC classes by using methods of the virtual moduli cycle. However a few properties of QC classes may potentially require monotonicity, notably Theorem 1.15 . 
Remark 1.8 We make no claim for uniqueness of these classes, as there are not enough axioms here. We are missing something like a normalization axiom (see for example Milnor and Stasheff [21]). It would be interesting to know if one can find a suitable substitute.

\subsection{Generalized Seidel representation}

For the following discussion we consider the based loop space $\Omega \operatorname{Ham}(M, \omega)$. This is a topological group with product induced by the product in $\operatorname{Ham}(M, \omega)$, there is an induced product on homology, the Pontryagin product, giving $H_{*}(\Omega \operatorname{Ham}(M, \omega))$ the structure of a ring. Let $f: B \rightarrow L$ Ham be a map from a smooth oriented closed $k$-manifold, and $P_{f}$ the induced fibration. Define

$$
\Psi(B, f) \equiv c_{k}^{q}\left(P_{f}\right)(B) \in Q H_{2 n+k}(M) .
$$

We will show that this induces a map

$$
\Psi: H_{*}(\Omega \operatorname{Ham}(M, \omega), \mathbb{Q}) \rightarrow Q H_{2 n+*}(M) .
$$

The Whitney sum formula (Axiom 3) will imply that $\Psi$ is a graded ring homomorphism.

Theorem 1.9 Let $(M, \omega)$ be a closed, monotone symplectic manifold of dimension 2n. There is a natural graded ring homomorphism

$$
\Psi: H_{*}(\Omega \operatorname{Ham}(M, \omega), \mathbb{Q}) \rightarrow Q H_{*+2 n}(M),
$$

where the product on the right is the quantum product.

In Section 3 and Section 4 we will describe these constructions and results in detail and give some computations and applications.

\subsection{Applications from Section 5}

Given a map

$$
f: B \rightarrow L \text { Ham } \quad \text { or } \quad f: B \rightarrow Q=\left(L \operatorname{Ham}(M, \omega) \times S^{\infty}\right) / S^{1},
$$

where $B$ is as before, we call

$$
L^{+}(f) \equiv \max _{b \in B} L^{+}\left(\gamma_{b}\right),
$$

the positive max-length measure of $f$, where $\gamma_{b}$ is either the loop $f(b) \in L$ Ham or the $S^{1}$ equivariant loop $f(b) \in Q$. More precisely, in the second case let $q: L \mathrm{Ham} \times S^{\infty} \rightarrow$ 
$Q$ denote the $S^{1}$ quotient map. Then $f(b)=q\left(\gamma_{b}, s_{b}\right)$ for some $\left(\gamma_{b}, s_{b}\right)$ and any two choices are related by an action of $S^{1}$ and hence the corresponding loops $\gamma_{b}$ have the same length.

We define the virtual index of a one parameter subgroup $\gamma: S^{1} \rightarrow \operatorname{Ham}(M, \omega)$ by

$$
I(\gamma)=\sum_{\substack{1 \leq i \leq n \\ k_{i} \leq-1}} 2\left(\left|k_{i}\right|-1\right),
$$

where $k_{i}$ are the weights of $\gamma$ at the max level set $F_{\max }$ of $H$ (see (1)).

We shall see in Section 2.3 that a map $\widehat{f}: Y \rightarrow \operatorname{Ham}(M, \omega)$, where $q: Y \rightarrow B$ is a principal $S^{1}$ bundle induces a cycle $f: B \rightarrow Q$.

Theorem 1.10 Let $(M, \omega)$ be a compact monotone symplectic manifold and let $\hat{f}: Y \rightarrow \operatorname{Ham}(M, \omega)$ be equivariant with respect to a right action by $\gamma: S^{1} \rightarrow$ $\operatorname{Ham}(M, \omega)$ on $\operatorname{Ham}(M, \omega)$, such that $I(\gamma)=\operatorname{dim} B$ and $e^{1 / 2 \operatorname{dim} B} \neq 0$ (or $\operatorname{dim} B=$ 0 ) where $e$ is the Euler class of the $S^{1}$ bundle $Y \rightarrow B$. Then the induced cycle $f: B \rightarrow Q$ is essential, ie doesn't vanish in the oriented bordism group $\operatorname{Bord}_{I(\gamma)}(Q)$, and moreover it minimizes the positive max-length measure in its bordism class.

Remark 1.11 In [25] Ustilovsky gives a formula for the Hessian, ie the "second variation formula" for the Hofer length functional and its positive and negative variants. We might try to define the index of a Hofer geodesic $\gamma$ to be the dimension of the maximum subspace of the tangent space to $\gamma$ (in $L$ Ham) on which the corresponding Hessian for the positive Hofer length functional is negative definite. This index could well be infinite as we are working on the loop space of the infinite dimensional space $\operatorname{Ham}(M, \omega)$. However, Theorem 1.10 suggests that at least for the geodesic coming from a circle action that satisfies hypotheses of the theorem the index must be finite. The heuristic argument for this, as well as for necessity of the virtual index condition of the theorem, is the following. Up to the action of $S^{1}$, all the loops in the image $f(B)$ are of the form $\hat{f}(y) \circ \gamma$ for $y \in Y$, by our assumption that $\hat{f}: Y \rightarrow \operatorname{Ham}(M, \omega)$ is $S^{1}$ equivariant. Since the Hofer metric is bi-invariant all these loops have the same index as $\gamma$. Moreover, we should get a certain vector bundle over the image $f(B)$ whose fiber over the equivariant loop $f(b), b \in B$, is "the" maximum negative definite subspace of the tangent space to $f(b)$, with respect to the corresponding Hessian. This is slightly wrong as there is no way to canonically pick out this negative definite subspace. However, we can fix such a subspace of the tangent space at $\gamma$ and then use the fact that all the other loops are translates of $\gamma$ of the form $\hat{f}(y) \circ \gamma$ up to the action of $S^{1}$ to construct this bundle locally and glue to get a global bundle. Let's call this ND bundle. If the rank of this ND bundle, given by the index, is bigger than 
$\operatorname{dim} B$ we can push the zero section off of itself and then "exponentiate" to produce a deformation of the cycle $f: B \rightarrow Q$ which reduces the max length measure; an apparent contradiction. On the other hand if the index is equal to $\operatorname{dim} B$, then there is an obstruction to reducing the max length measure by such a local move coming from the Euler class of the ND bundle. Lastly, if the index is strictly less than $\operatorname{dim} B$ there is still an obstruction coming from the Euler class but it is no longer in the top cohomology of $B$. Therefore while the cycle $f: B \rightarrow Q$ may minimize the max-length measure locally and maybe even in its homotopy class it, it may be unreasonable to hope that it is minimizing in the entire bordism class.

Remark 1.12 This heuristic argument suggests that a necessary condition for minimality of $f: B \rightarrow Q$ above is that the index is equal to $\operatorname{dim} B$. This condition is local; on the other hand the conclusion of Theorem 1.10 is global. Nevertheless, to prove it we compute the "leading order" contribution to the top quantum characteristic class of the associated bundle $p: P_{f} \rightarrow B$, in terms of the Euler class of a vector bundle analogous to the ND bundle above. A bit more precisely, this bundle will be an obstruction bundle for a certain moduli space of holomorphic curves (cf Section 5.1).

These remarks motivate the questions.

Question 1.13 Is the index of $\gamma$ as defined above finite? Do the index of $\gamma$ and the virtual index of $\gamma$ coincide? We will say a bit on this topic in a planned sequel [23].

Example 1.14 (for Theorem 1.10) Consider the Lie group homomorphism $\widehat{f}: S^{3} \rightarrow$ $\operatorname{Ham}\left(\mathbb{C P}^{n}, \omega\right)$, given by $s \cdot\left(\left[z_{0}, z_{1}, \ldots, z_{n}\right]\right)=\left[s\left(z_{0}, z_{1}\right), \ldots, z_{n}\right]$ for all $s \in S^{3}=S U(2),\left[z_{0}, \ldots, z_{n}\right] \in \mathbb{C P}^{n}$.

We can form an $S^{1}$ bundle $h: S^{3} \rightarrow S^{2}$ by taking the quotient of $S U(2)$ by the right action of the diagonal $S^{1}$ subgroup $\theta \mapsto\left(e^{i \theta}, e^{-i \theta}\right)$. If we take $\gamma: S^{1} \rightarrow$ $\operatorname{Ham}\left(\mathbb{C} \mathbb{P}^{n}, \omega\right)$ to be the subgroup

$$
e^{i \theta} \cdot\left[z_{0}, z_{1}, \ldots, z_{n}\right]=\left[e^{i \theta} z_{0}, e^{-i \theta} z_{1}, z_{2}, \ldots, z_{n}\right],
$$

acting on $\operatorname{Ham}\left(\mathbb{C} \mathbb{P}^{n}, \omega\right)$ on the right then the map $\hat{f}$ is $S^{1}$ equivariant for the two actions. The weights of $\gamma$ at the maximum $\max =[1,0,0, \ldots]$ of the generating function $H$ are $-2,-1,-1, \ldots$ and so $I(\gamma)=2$. Thus, by Theorem 1.10 the associated cycle

$$
f_{h}: S^{2} \rightarrow Q
$$

is essential and minimizes the max-length measure in its bordism class. 
But there is another cycle we can assign to $\hat{f}$. This is the cycle

$$
f: S^{2} \rightarrow \Omega \operatorname{Ham}(M, \omega)
$$

obtained from $\hat{f}: S^{3} \rightarrow \operatorname{Ham}\left(\mathbb{C} \mathbb{P}^{n}, \omega\right)$ by slicing $S^{3}$ into a bouquet of circles (cf Example 2.8). One can show that this cycle is essential by more elementary methods (cf Kedra-McDuff [9] and Reznikov [22]), but these arguments do not show that it minimizes the max-length measure.

The only non trivial characteristic class of $P_{f}$ is $c_{2}^{q}\left(P_{f}\right)$. Computing this directly is difficult, but we may use the following theorem proved in Section 5.3.

Theorem 1.15 Let $(M, \omega)$ be a spherically monotone, compact symplectic manifold, $\widehat{f}: S^{2 k+1} \rightarrow \operatorname{Ham}(M, \omega)$ a smooth map, and

$$
f_{h}: \mathbb{C P}^{k} \rightarrow Q, \quad f: S^{2 k} \rightarrow \Omega \operatorname{Ham}(M, \omega)
$$

obtained from $\hat{f}$ as in Example 2.8. Then the only possibly nontrivial characteristic classes of $P_{f_{h}}$ and $P_{f}$ in degree other than 0 are the top characteristic classes $c_{2 k}^{q}\left(P_{f_{h}}\right)$, $c_{2 k}^{q}\left(P_{f}\right)$ and

$$
\Psi\left(f_{h}, \mathbb{C P}^{k}\right)=\Psi\left(f, S^{2 k}\right) \in Q H_{2 n+2 k}(M)
$$

Note that $c^{q}\left(P_{f_{h}}\right)$ and $c^{q}\left(P_{f}\right)$ are computed via PGW invariants of two topologically very different fibrations, as $f$ and $f_{h}$ are not even homologous in $Q$. So there is no obvious apriori reason for (6) to hold. Using this as well as Proposition 5.3 and Example 5.4 we deduce that for our $f: S^{2} \rightarrow \Omega \operatorname{Ham}(M, \omega)$,

$$
\Psi\left(S^{2}, f\right)=[-\mathrm{pt}] \otimes q^{-m_{\max }} t^{H_{\max }}+\text { lower } t \text {-order terms } \in Q H_{2 n+2}\left(\mathbb{C P}^{n}\right),
$$

where $m_{\max }=\sum_{i} k_{i}=-2-(n-1)$ is the sum of the weights at the max and $H_{\max }$ is the maximum of $H$. Using the above and Proposition 5.1 we can deduce the following.

Corollary 1.16 The above map $f: S^{2} \rightarrow \Omega \operatorname{Ham}(M, \omega)$ is minimal in its rational homology class for the max-length measure.

Remark 1.17 The crucial part of the above calculation is that

$$
\hat{f}: S U(2) \rightarrow \operatorname{Ham}\left(\mathbb{C P}^{n}, \omega\right)
$$

is $S^{1}$-equivariant in an appropriate way, and so we may apply Theorem 1.10. One may try to extend the calculation by taking

$$
\widehat{f}: S U(n) \rightarrow \operatorname{Ham}\left(\mathbb{C} \mathbb{P}^{n-1}, \omega\right),
$$


and consider some associated cycle $f_{h}: S U(n) / S^{1} \rightarrow Q$. However, the nonvanishing condition on the Euler class in Theorem 1.10, $e^{1 / 2(\operatorname{dim} S U(n)-1)} \neq 0$ will never be satisfied because of the topology of the group $S U(n)$, according to McDuff [13]. There may of course be other examples, possibly not even coming from Lie group actions.

\subsection{Some questions}

Question 1.18 Does $f: S^{2} \rightarrow \Omega \operatorname{Ham}(M, \omega)$ remain minimal under the iterated Pontryagin product, with respect to the max-length measure, ie is

$$
f^{k}:\left(S^{2}\right)^{k} \rightarrow \Omega \operatorname{Ham}(M, \omega)
$$

minimal in its homology class?

A computation using Theorem 1.9 shows that the lower bounds coming from characteristic classes (Proposition 5.1) would grow to infinity but it is not clear if they stay sharp.

The following theorem is a slight reformulation of McDuff-Slimowitz [18].

Theorem 1.19 Let $\gamma: S^{1} \rightarrow \operatorname{Ham}(M, \omega)$ be a Hamiltonian circle action generated by a Morse Hamiltonian $H$. Suppose $\gamma$ is a local minimum of the Hofer length functional. Then it is a global minimum in its homotopy class.

Proof (sketch) It is well known that the max, min level sets of a Hamiltonian circle action are connected. Thus, since $H$ is Morse there is a unique max and min. Consider the following theorem.

Theorem 1.20 (McDuff-Lalonde [11]) Let $H_{t}, t \in[0,1]$ be a Hamiltonian defined on any symplectic manifold $M$, and $\gamma=\phi_{t}$ the corresponding isotopy. Assume that each fixed extremum of $H_{t}$ is isolated among the set of fixed extrema. If $\gamma$ is a stable geodesic (ie a local minimum of the length functional) there exist at least one fixed minimum $p$ and one fixed maximum $P$ at which the differential of the isotopy has no non constant closed trajectory in time less than 1 .

In our case this says that when $\gamma$ is a local minimum of the Hofer length functional and is generated by a Morse Hamiltonian the linearized flow at max and min corresponding to $\gamma$ has no nonconstant periodic orbits with period less than 1 . This condition is called semifree at max and min. On the other hand this puts us in position to apply the following theorem. 
Theorem 1.21 (McDuff-Tolman [19]) Let $\gamma$ be a Hamiltonian circle action with semifree maximal fixed point set and generating function $H$. Then there are classes $a_{B} \in H_{*}(M)$ such that:

$$
S(\gamma)=\left[F_{\max }\right] \otimes q^{-m_{\max }} t^{H_{\max }}+\sum_{B \in H_{2}^{S} \mid \omega(B)>0} a_{B} \otimes q^{-m_{\max }-c_{1}(B)} t^{H_{\max }-\omega_{B}}
$$

Here $S$ is the Seidel representation of (2), $H_{\max }$ denotes the maximum value of $H$ and $F_{\max }$ denotes the max level set. This expression implies that the positive Hofer length of the loop $\gamma$ is bounded below by $H_{\max }$ (cf Proposition 5.1). Reversing $\gamma$ and applying the same theorem, we similarly get that the negative Hofer length of $\gamma$ is bounded below by $-H_{\min }$. Together this implies the Hofer length of $\gamma$ is bounded below by $H_{\max }-H_{\min }$.

Question 1.22 Can the condition on $H$ being Morse in Theorem 1.19 be dropped or relaxed?

We can think of Theorem 1.19 and Theorem 1.10 as local to global rigidity type of phenomena in $\operatorname{Ham}(M, \omega)$. One may wonder to what extent this can be extended. One question which motivated this paper is the following.

Question 1.23 Let $G$ be a closed $k$-dimensional Lie group and $h: G \rightarrow \operatorname{Ham}(M, \omega)$ a Lie group homomorphism (perhaps with finite kernel). Suppose $h$ is a local minimum for a "natural volume functional" induced by the Hofer metric on $\operatorname{Ham}(M, \omega)$. Is $h$ necessarily a global minimum in its homotopy class? Homology class?

There are a few natural notions of volume in a Finsler manifold; one that is often used is the Hausdorff $k$-measure but it may not be the easiest to work with. We refer the reader to Álvarez and Thompson [1] for a discussion of these notions.

Acknowledgements This is part of the author's doctoral research at SUNY Stony Brook. I would like to thank my advisor Dusa McDuff for her patience and support both moral and mathematical, and numerous suggestions in writing this paper. I am grateful to Blaine Lawson, Dennis Sullivan and Aleksey Zinger for many useful discussions and comments and to an anonymous referee for careful reading and some keen observations and comments. 


\section{Preliminaries and setup}

In this section we describe constructions of certain natural fibrations, and of Parametric Gromov-Witten invariants defined on the total spaces of these fibrations.

Let $Q$ be the Borel $S^{1}$ quotient of $L$ Ham, $Q=\left(L H a m \times S^{\infty}\right) / S^{1}$, where the action of $S^{1}$ on $S^{\infty}$ is by multiplication by $e^{i \tau}$, for $\tau \in S^{1}$ and on $L$ Ham by $(\tau \cdot \gamma)(\theta)=\gamma(\theta+\tau)$. Let $q$ denote the quotient map

$$
q: L \operatorname{Ham} \times S^{\infty} \rightarrow Q .
$$

\subsection{Fibrations over LHam and $Q$}

There is a natural fibration over LHam:

$$
\tilde{p}: U \rightarrow L \mathrm{Ham}
$$

where

$$
U=L \operatorname{Ham} \times M \times D_{0}^{2} \cup L \operatorname{Ham} \times M \times D_{\infty}^{2} / \sim
$$

and the equivalence relation $\sim$ is: $(\gamma, x, 1, \theta)_{0} \sim\left(\gamma, \gamma_{\theta}(x), 1, \theta\right)_{\infty}$. Here, $(r, 2 \pi \theta)$ are polar coordinates on $D^{2}$, and $\gamma_{\theta}$ denotes the element of the loop $\gamma$ at time $\theta$. The orientation on $M \times D_{0}^{2}$ is taken to be the natural positive orientation and on $M \times D_{\infty}^{2}$ is taken to be negative. There is a natural $S^{1}$ action on $U$

$$
\tau \cdot(\gamma, x, r, \theta)_{0, \infty}=(\tau \cdot \gamma, x, r, \theta-\tau)_{0, \infty}
$$

where $\tau \in S^{1}$ and $(\tau \cdot \gamma)_{\theta}=\gamma_{\theta+\tau}$, ie the standard $S^{1}$ action on the loop space. It can be quickly checked that this is well defined under the equivalence relation $\sim$. Thus, the diagonal action $\rho$ of $S^{1}$ on $L \mathrm{Ham} \times S^{\infty}$ lifts to a diagonal action $\tilde{\rho}$ on the product fibration

$$
\tilde{p} \times \text { id: } U \times S^{\infty} \rightarrow L \operatorname{Ham} \times S^{\infty} .
$$

This gives a quotient bundle

$$
p: U^{S^{1}}=\left(U \times S^{\infty}\right) / S^{1} \rightarrow Q .
$$

The fiber $X_{q(\gamma, s)}$ of $U^{S^{1}}$ over $q(\gamma, s)$ (see (7)) is the total space of the Hamiltonian bundle $X_{\gamma}$ (cf (3)). We recall for the reader:

Definition 2.1 A Hamiltonian bundle is a bundle with symplectic fiber, whose transition maps are Hamiltonian. A Hamiltonian bundle map is a bundle map which preserves the Hamiltonian bundle structure. 
Remark 2.2 We show in Section 7 that the structure group of $\tilde{p}: U \rightarrow L$ Ham over the component containing the loop $\gamma$ may be reduced to the group $\mathcal{F}^{\gamma}$ of Hamiltonian bundle maps of the fiber $X_{\gamma}$, which are identity over $D_{0}^{2}$ and a neighborhood of $0 \in D_{\infty}^{2}$. A very similar description holds for the structure group of $p: U^{S^{1}} \rightarrow Q$, in particular it consists of certain Hamiltonian bundle maps. The groups $\mathcal{F}^{\gamma}$ are isomorphic for all $\gamma$ and we just refer to the groups as $\mathcal{F}$. As already mentioned in the Introduction and proved in Section 7, the space LHam is the classifying space for $\mathcal{F}$. (More precisely, the component of the loop $\gamma$ in $L$ Ham is the classifying space for $\mathcal{F}^{\gamma}$.) We call a fiber bundle $p: P \rightarrow B$, with fiber having the structure of a Hamiltonian fibration $\pi: X \rightarrow S^{2}$ and structure group $\mathcal{F}$ an $\mathcal{F}$-fibration. The structure group of the bundle pulled back from $p: U^{S^{1}} \rightarrow Q$ is also determined in Section 7 and it also consists of special Hamiltonian bundle maps. We will call both types of bundles simply by $\mathcal{F}$-fibration, where there can be no confusion.

\subsection{Families of symplectic forms on an $\mathcal{F}$-fibration}

Let $p: P \rightarrow B$ be an $\mathcal{F}$-fibration, in the sense of above remark. Fix an area form $\alpha$ on the base $S^{2}$ of $\pi: X \rightarrow S^{2}$ once and for all. Since the fibers $M$ are canonically oriented as symplectic manifolds and since the transition maps of $\pi: X \rightarrow S^{2}$ are Hamiltonian and hence preserve that orientation, this induces an orientation $\sigma$ on the fibers $X$ of $P$, which is again preserved by the structure group $\mathcal{F}$ of the bundle $P$. Thus, since $B$ is oriented $P$ inherits a well defined orientation.

Definition 2.3 Let $\pi: X \rightarrow S^{2}$ be a Hamiltonian fibration with fiber $(M, \omega)$. We say that a symplectic form $\Omega$ on $X$ is $\omega$-compatible if it extends $\omega$ on the fibers.

Let $\mathcal{A}$ consist of all $\omega$-compatible symplectic forms $\Omega$ on $X$ inducing the orientation $\sigma$ (note, the cohomology class of $\Omega$ is not fixed). Since $\mathcal{F}$ acts on $\mathcal{A}$, we have the associated bundle $k: K_{P} \rightarrow B$ with fiber $\mathcal{A}$.

Definition 2.4 Let $p: P \rightarrow B$ be an $\mathcal{F}$-fibration. A family of symplectic structures $\left\{\Omega_{b}\right\}$ on $P$ is called admissible if it is a section of $K_{P}$.

Lemma 2.5 The space of admissible families $\left\{\Omega_{b}\right\}$ on $p: P \rightarrow B$ is connected and nonempty.

Proof We show that the fiber $\mathcal{A}$ of the bundle $K$ is at least weakly contractible, ie has vanishing homotopy groups. It will follow from obstruction theory that the space of sections is connected and nonempty. 
Let $h: S^{k} \rightarrow \mathcal{A}$ be a continuous map. We denote $h(s)$ by $\Omega_{s}$. Let $\Omega_{0} \in \mathcal{A}$. The path

$$
\Omega_{t, s}=t \Omega_{0}+(1-t) \Omega_{s}, \quad t \in I=[0,1]
$$

may not lie in $\mathcal{A}$, as $\Omega_{t, s}$ may be degenerate for some $t$, so we will need to compensate. For $t, s \in I \times S^{k}$, let Hor ${ }^{t, s}$ denote the horizontal subbundle of $T X$ with respect to $\Omega_{t, s}$, ie Hor ${ }^{t, s}$ is the symplectic orthogonal to the vertical tangent bundle of $\pi: X \rightarrow S^{2}$.

Let $\Omega_{t, s}^{h}$ denote the horizontal part of $\Omega_{t, s}$, ie $\Omega_{t, s}^{h}$ is zero on the vertical subbundle of $T X$ and coincides with $\Omega_{t, s}$ on Hor ${ }^{t, s}$. Then

$$
\Omega_{t, s}^{h}=f_{t, s} \cdot \pi^{*}(\alpha), \text { where } f_{t, s}: X \rightarrow \mathbb{R} \text { is smooth. }
$$

Recall that $\alpha$ is the fixed area form on $S^{2}$. Set

$$
C=\left|\inf _{t, s \in I \times S^{k}}\left(\inf _{X} f_{t, s}\right)\right|+1
$$

and define

$$
\begin{aligned}
& \phi(t)= \begin{cases}0 & \text { if } t \in[0,1 / 3] ; \\
3(t-1 / 3) & \text { if } t \in[1 / 3,2 / 3] ; \\
1 & \text { if } t \in[2 / 3,1] ;\end{cases} \\
& \eta(t)= \begin{cases}3 t & \text { if } t \in[0,1 / 3] ; \\
1 & \text { if } t \in[1 / 3,2 / 3] ; \\
-3(t-2 / 3)+1 & \text { if } t \in[2 / 3,1] .\end{cases}
\end{aligned}
$$

Consider the following homotopy of the map $h$ :

$$
F(t, s)=\phi(t) \Omega+(1-\phi(t)) \Omega_{s}+\eta(t) C \pi^{*}(\alpha) .
$$

Then $F(1, x)$ is the constant map to $\Omega_{0}$ and $F(0, x)=h(x)$. Since that $\Omega_{s}$ and $\Omega$ induce the same orientation on $X, f_{0, s}, f_{1, s}>0$. Using this, it is clear that the form $F(t, x)$ is nondegenerate on $X$ for every $t, x$, and so $F(t, x)$ is a map into $\mathcal{A}$. Thus, all the homotopy groups of $\mathcal{A}$ vanish.

This discussion shows that we may choose an admissible family $\left\{\Omega_{b}\right\}$ on $P$ and moreover any two such families are deformation equivalent. We will now construct a special family that will be crucial in applications to the Hofer metric. As the first step we define a family of symplectic forms $\left\{\widetilde{\Omega}_{\gamma}^{\infty}\right\}$ on $L \mathrm{Ham} \times M \times D_{\infty}^{2}$ :

$$
\widetilde{\Omega}_{\gamma}^{\infty}(x, r, \theta)=\omega+d\left(\eta(r) H_{\theta}^{\gamma}\left(\gamma_{0}^{-1} x\right)\right) \wedge d \theta-\max _{x} H_{\theta}^{\gamma}(x) d \eta \wedge d \theta-\epsilon \cdot 2 r d r \wedge d \theta,
$$


for an $\epsilon>0$. (Recall that $M \times D_{\infty}^{2}$ has the negative orientation.) Here, $H_{\theta}^{\gamma}$ is the generating Hamiltonian for $\gamma^{-1}(0) \circ \gamma$, normalized so that

$$
\int_{M} H_{\theta}^{\gamma} \omega^{n}=0
$$

for all $\theta$, and $\eta:[0,1] \rightarrow[0,1]$ is a smooth function satisfying

$$
0 \leq \eta^{\prime}(r)
$$

and

$$
\eta(r)= \begin{cases}1 & \text { if } 1-\delta \leq r \leq 1, \\ r^{2} & \text { if } r \leq 1-2 \delta,\end{cases}
$$

for a small $\delta>0$. The last 2 terms are needed to make the sum nondegenerate. The following geometric notion will be important to us for tying Hofer geometry with geometry of holomorphic curves.

Definition 2.6 The area of a Hamiltonian fibration $\pi: X \rightarrow S^{2}$ or $\pi: X \rightarrow D^{2}$, together with an $\omega$-compatible symplectic form $\Omega$ is defined by:

$$
\operatorname{area}(X, \Omega)=\operatorname{Vol}(X, \Omega) / \operatorname{Vol}(M, \omega)=\frac{\int_{X} \Omega^{n+1}}{(n+1) \int_{M} \omega^{n}} .
$$

The area of $\widetilde{\Omega}_{\gamma}^{\infty}$ on $M \times D_{\infty}^{2}$ is constructed to be $L^{+}(\gamma)+\epsilon$.

By definition of $\sim,(x, \theta)_{0} \mapsto\left(\gamma_{\theta}(x), \theta\right)_{\infty}$. Thus,

$$
\frac{\partial}{\partial \theta} \mapsto\left(\gamma_{0}\right)_{*}\left(X_{H_{\gamma}^{\theta}}\right)+\frac{\partial}{\partial \theta}, \quad \frac{\partial}{\partial x} \mapsto\left(\gamma_{\theta}\right)_{*}\left(\frac{\partial}{\partial x}\right) \quad \text { and } \quad \frac{\partial}{\partial r} \mapsto-\frac{\partial}{\partial r} \text {. }
$$

It follows that the gluing relation $\sim$ pulls back the form $\widetilde{\Omega}_{\gamma}^{\infty}$ to the form

$$
\widetilde{\Omega}_{\gamma}^{0}=\omega+\epsilon \cdot 2 r d r \wedge d \theta
$$

on the neighborhood of the boundary $M \times \partial D_{0}^{2}$, which extends to the form $\widetilde{\Omega}_{\gamma}^{0}$ on $M \times D_{0}^{2}$ with area $\epsilon$. Then $\left\{\widetilde{\Omega}_{\gamma}\right\}$ on $U$ is given by gluing

$$
\left(L \operatorname{Ham} \times M \times D_{0}^{2}, \widetilde{\Omega}_{\gamma}^{0}\right) \cup\left(L \operatorname{Ham} \times M \times D_{\infty}^{2}, \widetilde{\Omega}_{\gamma}^{\infty}\right) / \sim .
$$

The area of each fiber is

$$
\operatorname{area}\left(X_{\gamma}, \widetilde{\Omega}_{\gamma}\right)=L^{+}(\gamma)+2 \epsilon \text {. }
$$

We pull back the family $\left\{\widetilde{\Omega}_{\gamma}\right\}$ on $U$ to a family $\left\{\widetilde{\Omega}_{(\gamma, s)}\right\}$ on $U \times S^{\infty}$ via projection to $U$. The $S^{1}$ action $\tilde{\rho}$ does not act by a symplectomorphism from the fiber $X_{(\gamma, s)}$ to 
the fiber $X_{(\tau \cdot \gamma, \tau \cdot s)}$. We can fix this problem by averaging. Define a family $\left\{\widetilde{\Omega}_{(\gamma, s)}^{S^{1}}\right\}$ on $U \times S^{\infty}$ by

$$
\widetilde{\Omega}_{(\gamma, s)}^{S^{1}}=\frac{1}{2 \pi} \int_{S^{1}} \tilde{\rho}(\tau)^{*} \widetilde{\Omega}_{(\theta \cdot \gamma, \theta \cdot s)} d \tau .
$$

On $\left(L \operatorname{Ham} \times S^{\infty}\right) \times M \times D_{\infty}^{2}$ this form is

$$
\begin{aligned}
\widetilde{\Omega}_{(\gamma, s)}^{S^{1}}=\omega-\max _{x} H_{\theta}^{\gamma}(x) d \eta \wedge d \theta-\epsilon \cdot & 2 r d r \wedge d \theta \\
& +\frac{1}{2 \pi} \int_{S^{1}}\left(d\left(\eta(r) H_{\theta}^{\gamma}\left(\gamma(\tau)^{-1} x\right)\right) \wedge d \theta\right) d \tau .
\end{aligned}
$$

It follows that each $\widetilde{\Omega}_{(\gamma, s)}^{S^{1}}$ is symplectic and

$$
\operatorname{area}\left(X_{(\gamma, s)}, \widetilde{\Omega}_{\gamma, s}^{S^{1}}\right)=L^{+}(\gamma)+2 \epsilon
$$

as before. Thus, the family $\widetilde{\Omega}_{(\gamma, s)}^{S^{1}}$ on $U \times S^{\infty}$ passes down to a family $\left\{\Omega_{b}\right\}$ on the quotient bundle $p: U^{S^{1}} \rightarrow Q$ with

$$
\operatorname{area}\left\{\Omega_{b}\right\}=L^{+}(\gamma)+2 \epsilon .
$$

\subsection{Equivariant cycles in LHam}

Let $B$ be oriented compact and smooth. Up to homotopy, every cycle $f: B \rightarrow Q$ arises as follows. Let $g: Y \rightarrow B$ be a smooth principal $S^{1}$ bundle. And let $\hat{f}: Y \rightarrow$ $\operatorname{Ham}(M, \omega)$ be a map. Define

$$
o: Y \rightarrow L Y
$$

to be the map which sends $x \in Y$ to the loop $\gamma_{x}, \gamma_{x}(\theta)=x \cdot \theta$, also let $f^{\prime}: L Y \rightarrow$ $L$ Ham be the map induced by $\hat{f}: Y \rightarrow \operatorname{Ham}(M, \omega)$. Set $\tilde{f}=f^{\prime} \circ o$, then

$$
\tilde{f}: Y \rightarrow L \text { Ham }
$$

is $S^{1}$ equivariant. Let $c: Y \rightarrow S^{\infty}$ be an $S^{1}$ equivariant map. (The $S^{1}$ equivariant homotopy class of this map is uniquely determined.) Consider the product map

$$
\tilde{f} \times c: Y \rightarrow L \operatorname{Ham} \times S^{\infty} .
$$

This is again an $S^{1}$ equivariant map under the diagonal $S^{1}$ action and so induces a map on the quotients $f: B \rightarrow Q$, whose homotopy class is independent of the choice of the classifying map $c$.

Definition 2.7 We will call $f: B \rightarrow Q$ smooth, if it comes from a smooth map $\widehat{f}: Y \rightarrow \operatorname{Ham}(M, \omega)$. 
Clearly any map $f: B \rightarrow Q$ can be perturbed to be smooth.

Example 2.8 Let's apply the above construction to a map $\hat{f}: S^{2 k+1} \rightarrow \operatorname{Ham}(M, \omega)$. We can associate to it two cycles in $Q$, by slicing $S^{2 k+1}$ by circles in two different ways. The first cycle, $f_{h}: \mathbb{C P}^{k} \rightarrow Q$ is obtained from the Hopf fibration $h: S^{2 k+1} \rightarrow \mathbb{C P}^{k}$. The second $f: S^{2 k} \rightarrow Q$ is obtained from the trivial fibration pr: $S^{2 k} \times S^{1} \rightarrow S^{2 k}$ and the composition $\hat{f}_{2}=\hat{f} \circ t: S^{2 k} \times S^{1} \rightarrow \operatorname{Ham}(M, \omega)$, where

$$
t: S^{2 k} \times S^{1} \rightarrow S^{2 k+1}
$$

is any fixed degree 1 map. The maps $f$ and $f_{h}$ are not homologous since any such homology would project to a homology in $\mathbb{C P}^{\infty}$, for the classifying maps of the bundles pr: $S^{2 k} \times S^{1} \rightarrow S^{2 k}$ and $h: S^{2 k+1} \rightarrow \mathbb{C P}^{k}$.

Remark 2.9 Given a smooth map $f: B \rightarrow Q$ the pullback bundle $p_{f}: P_{f} \rightarrow B$ by $f$ of the bundle $p: U^{S^{1}} \rightarrow Q$ can be given the following tautological reformulation, which will be useful to us. The map $f$ comes from a smooth map $\hat{f}: Y \rightarrow \operatorname{Ham}(M, \omega)$ for a certain smooth oriented principal $S^{1}$ bundle $g: Y \rightarrow B$. This induces a map $\tilde{f}: Y \rightarrow L$ Ham, where $\tilde{f}$ is as in (10). Consider the pullback bundle

$$
p_{\tilde{f}}: P_{\tilde{f}} \rightarrow Y
$$

by $\tilde{f}$ of the bundle $\tilde{p}: U \rightarrow L$ Ham. In other words

$$
P_{\tilde{f}}=\left(Y \times M \times D_{0}^{2}\right) \cup\left(Y \times M \times D_{\infty}^{2}\right) / \sim
$$

where $(y, x, 1, \theta)_{0}$ is equivalent to $\left(y, \widetilde{f}_{t, \theta}(x), 1, \theta\right)_{\infty}$. This is a smooth bundle with the pullback of the $S^{1}$-action $\tilde{\rho}$ on $U$ given by

$$
\theta^{\prime} \cdot(y, x, r, \theta)_{0, \infty}=\left(\theta^{\prime} \cdot y, x, r, \theta-\theta^{\prime}\right)_{0, \infty} .
$$

The quotient by the $S^{1}$ action on this bundle is the bundle $p_{f}: P_{f} \rightarrow B$. Thus, when $f: B \rightarrow Q$ is smooth the bundle $p_{f}: P_{f} \rightarrow B$ and the family $\left\{f^{*}\left(\Omega_{b}\right)\right\}$ of symplectic forms on this bundle are smooth.

\subsection{Natural embeddings into an $\mathcal{F}$-fibration}

Now, let $f: B \rightarrow Q$ be as usual, $\tilde{f}: Y \rightarrow L$ Ham the associated $S^{1}$-equivariant map (cf (10)) and consider the associated fibration $P_{\tilde{f}}$ (cf (11)). There are natural embeddings

$$
\tilde{I}_{0, \infty}: Y \times M \rightarrow Y \times M \times D_{0, \infty}^{2}
$$


given by including into the fiber over $0 \in D_{0, \infty}^{2}$ and thus induced embeddings

$$
\tilde{I}_{0, \infty}: Y \times M \rightarrow P \tilde{f} .
$$

These maps are $S^{1}$ equivariant under the action of $\tilde{\rho}$ (cf (12)) and hence there are induced embeddings $I_{0, \infty}: B \times M \rightarrow P_{f}$, which be used later.

A special case If we consider $Q$ as $L$ Ham bundle over $\mathbb{C} P^{\infty}$, there is a natural map $i_{*}: H_{*}(L \mathrm{Ham}) \rightarrow H_{*}^{S^{1}}(L \mathrm{Ham})$ induced by inclusion of the fiber. Given a cycle $f^{\prime}: B \rightarrow L$ Ham, the bundle $P_{f}$ induced by the cycle $f=i \circ f^{\prime}: B \rightarrow Q$ can be easily seen to be isomorphic to the pullback by $\tilde{f}$ of the bundle $U$ over $L$ Ham, ie

$$
P_{f} \simeq\left(B \times M \times D_{0}^{2}\right) \cup\left(B \times M \times D_{\infty}^{2}\right) / \sim
$$

where for $(b, x, \theta)_{0}$ in the boundary of $B \times M \times D_{0}^{2},(b, x, \theta)_{0} \sim\left(b, f_{b, \theta}^{\prime}(x), \theta\right)_{\infty}$, and the embeddings

$$
I_{z}: B \times M \rightarrow P_{f},
$$

defined above are now defined for all $z \in S^{2}$. (This embedding is only well defined up to isotopy for $z$ in the equator $\partial D_{0, \infty}^{2} \in S^{2}$.)

The following subsection essentially sets up for Section 5.1 and its reading may be postponed until then. On the other hand, it may help to clarify the above constructions.

\subsection{Example of an $\mathcal{F}$-fibration}

Suppose now we have a map $\hat{f}: Y \rightarrow \operatorname{Ham}(M, \omega)$, where $q: Y \rightarrow B$ is an oriented principal $S^{1}$ bundle. Suppose further that the map $\hat{f}$ is $S^{1}$ equivariant with respect to the $S^{1}$ action on $Y$ and $S^{1}$ action on $\operatorname{Ham}(M, \omega)$ corresponding to the right action by a subgroup $\gamma: S^{1} \rightarrow \operatorname{Ham}(M, \omega)$ on $\operatorname{Ham}(M, \omega)$. Let us understand the fibration $P_{f}$ for the induced map

$$
f: B \rightarrow Q
$$

First, we can identify $X_{\gamma}$ with $S^{3} \times S^{1} M$, where $S^{1}$ acts diagonally on $S^{3} \times M$ by

$$
e^{2 \pi i \theta} \cdot\left(z_{1}, z_{2} ; x\right)=\left(e^{-2 \pi i \theta} z_{1}, e^{-2 \pi i \theta} z_{2} ; \gamma\left(e^{2 \pi i \theta}\right) x\right),
$$

using complex coordinates on $S^{3}$. To see this, write $\left[z_{1}, z_{2} ; x\right]$ for the equivalence class of the point $\left(z_{1} / r, z_{2} / r ; x\right) \in S^{3} \times M$, where $r$ is the norm of $\left(z_{1}, z_{2}\right)$. We identify $D_{0} \times M$ with $\{[1, z ; x]:|z| \leq 1, x \in M\}$ naturally and $D_{\infty} \times M$ with $\{[z, 1 ; x]:|z| \leq 1, x \in M\}$ via the orientation reversing reflection. The gluing map is then

$$
\left[1, e^{2 \pi i \theta} ; x\right] \sim\left[e^{-2 \pi i \theta}, 1 ; \gamma\left(e^{2 \pi i \theta}\right) x\right]
$$


consistent with the previous definition. There is an $S^{1}$ action $\beta$ on $X_{\gamma}$ given by

$$
e^{2 \pi i \theta^{\prime}} \cdot\left[z_{1}, z_{2} ; x\right]=\left[z_{1}, e^{2 \pi i \theta^{\prime}} z_{2} ; x\right] .
$$

Lemma 2.10 The bundle $p_{f}: P_{f} \rightarrow B$ is isomorphic to the bundle $h: Y \times_{S^{1}} X_{\gamma} \rightarrow B$, where $S^{1}$ is acting by $\beta$ on $X_{\gamma}$.

Proof Let $\tilde{f}: Y \rightarrow L$ Ham be as above (see (10)) so

$$
(\tilde{f}(y)=\hat{f}(y) \circ \gamma): S^{1} \rightarrow \operatorname{Ham}(M, \omega) .
$$

We have

$$
P_{\tilde{f}}=Y \times M \times D_{0}^{2} \cup Y \times M \times D_{\infty}^{2} / \sim
$$

where

$$
(y, x, 1, \theta)_{0} \sim\left(y, \hat{f}(y) \circ \gamma_{\theta}(x), 1, \theta\right)_{\infty} .
$$

In coordinates we have

$$
M \times D_{0}^{2} \cup Y \times M \times D_{\infty}^{2} / \sim
$$

where $(y, x, 1, \theta)_{0} \sim\left(y, \gamma_{\theta}(x), 1, \theta\right)_{\infty}$. There is a map $k: P_{\tilde{f}} \rightarrow Y \times X_{\gamma}$ defined as follows:

$$
\begin{aligned}
k(y, x, r, \theta)_{\infty} & =\left(y, \hat{f}(y)^{-1}(x), r, \theta\right)_{\infty} \\
k(y, x, r, \theta)_{0} & =(y, x, r, \theta)_{0} .
\end{aligned}
$$

This is a well defined bundle map, as is shown by the following diagram:

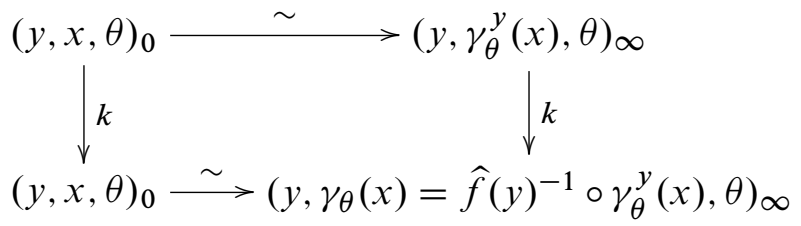

We have the $S^{1}$ action on $Y \times X_{\gamma}$ given by

$$
\begin{aligned}
\theta^{\prime} \cdot(y, x, r, \theta)_{0} & =\left(y \cdot \theta^{\prime}, x, r, \theta-\theta^{\prime}\right)_{0} \\
\theta^{\prime} \cdot(y, x, r, \theta)_{\infty} & =\left(y \cdot \theta^{\prime}, \gamma^{-1}\left(\theta^{\prime}\right) x, r, \theta-\theta^{\prime}\right)_{\infty} .
\end{aligned}
$$

It is now not hard to check that the map $k$ is $S^{1}$ equivariant with respect to the action $\tilde{\rho}(\mathrm{cf}(12))$ and the action given in (15)-(16). Finally, we conclude that

$$
P_{f} \simeq Y \times_{S^{1}} X_{\gamma}
$$


2.5.1 An admissible family of symplectic forms on $P_{f}$ Suppose $P_{f}$ is as in Lemma 2.10. Using this lemma we can put an admissible family $\left\{\Omega_{b}\right\}$ on $p: P_{f} \rightarrow B, b \in B$ and a compatible family of almost complex structures $\left\{J_{b}\right\}$ as follows. Let $\alpha$ be the standard contact form on the unit sphere $S^{3}$, normalized so that $d \alpha=h^{*} \tau$, where $h: S^{3} \rightarrow S^{2}$ is the Hopf map and $\tau$ is a standard area form on $S^{2}$ with area 1. If $H: M \rightarrow \mathbb{R}$ denotes the normalized Hamiltonian generating $\gamma$, the closed 2-form

$$
\omega-(\max H+\epsilon) d \alpha+d(H \alpha)
$$

on $S^{3} \times M$ descends to a form $\widetilde{\omega}$ on $S^{3} \times{ }_{S^{1}} M$, which is symplectic for an $\epsilon>0$. Let $J$ be any $S^{1}$-invariant almost complex structure on $M$ and $J_{0}$ the standard $S^{1}$ invariant complex structure on $\mathbb{C}^{2}$. Then $J \times J_{0}$ is also $S^{1}$-invariant, and its restriction to $S^{3}$ preserves the contact planes $\operatorname{ker} \alpha$. It is not hard to see that $J \times J_{0}$ descends to an almost complex structure $\widetilde{J}$ on the quotient $X_{\gamma}$ which coincides with $J$ on the fibers $M$. By construction, if $J$ is compatible with $\omega$, then $\widetilde{J}$ is compatible with $\widetilde{\omega}$. The form $\widetilde{\omega}$ and the complex structure $\widetilde{J}$ are invariant under the $S^{1}$ action $\beta$ on $X_{\gamma}$ and therefore give rise to a family $\left\{\Omega_{b}\right\}$ and a compatible family $\left\{J_{b}\right\}$ on $P_{f}=Y \times_{S^{1}} X_{\gamma}$.

\subsection{PGW invariants of an $\mathcal{F}$-fibration}

Let $p: P \rightarrow B$ be an $\mathcal{F}$-fibration.

Definition 2.11 We call a family $\left\{J_{b}\right\}$ of fiberwise $\left\{\Omega_{b}\right\}$-compatible complex structures $\pi$-compatible if $\pi:\left(X_{b}, J_{b}\right) \rightarrow\left(S^{2}, j\right)$ is holomorphic for each $b$ and each $J_{b}$ preserves the $\Omega_{b}$-orthogonal subspaces of $T X_{b}$, for some admissible family $\left\{\Omega_{b}\right\}$ on $P$.

Let $\left\{J_{b}\right\}$ be a $\pi$-compatible family of almost complex structures. Consider the following moduli space

$$
\mathcal{M}_{0}^{*}\left(P, A,\left\{J_{b}\right\}\right)=\{\text { pairs }(u, b)\}
$$

where

- $b \in B^{k}$.

- $u$ is a $J_{b}$-holomorphic, simple curve $u:\left(S^{2}, j\right) \rightarrow X_{b} \subset P$ representing class $A \in j_{*}\left(H_{2}^{\text {sect }}(X)\right) \subset H_{2}(P)$, where $H_{2}^{\text {sect }}(X)$ are section classes and $j_{*}$ is induced by inclusion of fiber. (The subspace $j_{*}\left(H_{2}^{\text {sect }}(X)\right)$ is unambiguous, since the structure group $\mathcal{F}$ preserves section classes of $X$.) 
An element of the above moduli space will be called loosely a fiber holomorphic curve. For details of the following discussion see for example McDuff and Salamon [17, Sections 6.7, 8.4] or Buse [2]. Given an element $(u, b)$ of the moduli space $\mathcal{M}_{0}^{*}\left(P, A,\left\{J_{b}\right\}\right)$ there is the associated real linear Cauchy-Riemann operator

$$
D_{u, b}:\left\{\xi \in \Omega^{0}\left(S^{2}, u^{*} T P\right) \mid d p(\xi) \equiv \mathrm{const}\right\} \rightarrow \Omega^{0,1}\left(S^{2}, u^{*} T X_{b}\right)
$$

of index $2 n+k+2 c_{1}(A)$, where $c_{1}$ is the vertical Chern class of the fibration $p: P \rightarrow B$. A $\pi$-compatible family $\left\{J_{b}\right\}$ is called regular for $A$, if the operator $D_{u, b}$ is surjective for every tuple $(u, b)$, where $u \in \mathcal{M}_{0}^{*}\left(P, A,\left\{J_{b}\right\}\right)$. The set of regular $\pi$-compatible families for $A$ will be denoted by $\mathcal{J}_{\text {reg }}(A)$ and the set of all families by $\mathcal{J}$. From now on regular family $\left\{J_{b}\right\}$ always refers to a $\pi$-compatible regular family.

Lemma 2.12 (1) If $\left\{J_{b}\right\} \in \mathcal{J}_{\text {reg }}(A)$ then $\mathcal{M}^{*}\left(P, A ;\left\{J_{b}\right\}\right)$ is a smooth manifold of dimension

$$
\operatorname{dim} \mathcal{M}^{*}\left(P, A ;\left\{J_{b}\right\}\right)=2 n+k+2 c_{1}(A) .
$$

(2) The set $\mathcal{J}_{\text {reg }}(A)$ is of the second category in $\mathcal{J}$.

Suppose now we have an oriented smooth cobordism $C$ between $B_{1}, B_{2}$. Let $P_{C}$ be a symplectic fibration over $C$. We denote by $P_{i}$ the restriction of $P_{C}$ over $B_{i}$. Suppose we have regular families $\left\{J_{b}^{i}\right\}$ on $P_{i}$. Let $\left\{J_{b}^{C}\right\}$ be family on $P_{C}$ restricting to $\left\{J_{b}^{i}\right\}$ on $P_{i}$. We then have the corresponding moduli space

$$
\mathcal{M}^{*}\left(P_{C}, A ;\left\{J_{b}^{C}\right\}\right) \text {. }
$$

We again say that $\left\{J_{b}^{C}\right\}$ is regular if the associated Cauchy-Riemann operator is surjective. The space of regular families $\left\{J_{b}^{C}\right\}$ will be denoted by $\mathcal{J}_{\text {reg }}\left(A ;\left\{J_{b}^{1}\right\},\left\{J_{b}^{2}\right\}\right)$, and the space of all families by $\mathcal{J}\left(A ;\left\{J_{b}^{1}\right\},\left\{J_{b}^{2}\right\}\right)$.

Lemma 2.13 (1) If $\left\{J_{b}^{C}\right\}$ is regular $\mathcal{M}^{*}\left(P_{C}, A ;\left\{J_{b}^{C}\right\}\right)$ is a smooth oriented manifold with boundary

$$
\partial \mathcal{M}^{*}\left(P_{C}, A ;\left\{J_{b}^{C}\right\}\right)=\mathcal{M}\left(P_{2}, A,\left\{J_{b}^{1}\right\}\right)-\mathcal{M}^{*}\left(P_{1}, A,\left\{J_{b}^{2}\right\}\right) .
$$

(2) The set $\mathcal{J}_{\text {reg }}\left(A ;\left\{J_{b}^{1}\right\},\left\{J_{b}^{2}\right\}\right)$ is of the second category in $\mathcal{J}\left(A ;\left\{J_{b}^{1}\right\},\left\{J_{b}^{2}\right\}\right)$.

Let $\mathcal{M}_{0, l}^{*}\left(P, A,\left\{J_{b}\right\}\right)=\left\{\right.$ equivalence classes of tuples $\left.\left(u, z_{1} \ldots z_{l}\right)\right\}$,

where $u \in \mathcal{M}^{*}\left(P, A ;\left\{J_{b}\right\}\right)$ and $z_{1}, \ldots, z_{l}$ are pairwise distinct points in $S^{2}$. The equivalence relation is $\left(u, z_{1}, . ., z_{l}\right) \sim\left(u^{\prime}, z_{1}^{\prime}, \ldots, z_{l}^{\prime}\right)$ if there exists $\phi \in \operatorname{PSL}(2, \mathbb{C})$ s.t. $u^{\prime} \circ \phi=u$ and $\phi\left(z_{i}\right)=z_{i}^{\prime}$. 
For a regular family $\left\{J_{b}\right\}$, this is a manifold of dimension

$$
2 n+k+2 c_{1}(A)+2 l-6,
$$

where $k$ is the dimension of the base $B$. Consider the evaluation map

$$
\text { ev: } \mathcal{M}_{0, l}^{*}\left(P, A ;\left\{J_{b}\right\}\right) \rightarrow P^{l} .
$$

Similarly, we have evaluation maps ev ${ }^{C}: \mathcal{M}^{*}\left(P_{C}, A ;\left\{J_{b}^{C}\right\}\right) \rightarrow P_{C}$. For these maps to represent pseudocycles we need some conditions on $M$.

Proposition 2.14 Let $(M, \omega)$ be spherically monotone. Then the maps ev and ev $C$ above are pseudocycles for generic regular $\pi$-compatible families $\left\{J_{b}\right\}$ and $\left\{J_{b}^{C}\right\}$.

Proof Since we only consider curves which lie in the fibers of $p: P \rightarrow B$, any bubbles must lie in the fiber. Next note that a stable map into $\left(X_{b}, J_{b}\right)$, representing a section class of $\pi: X_{b} \rightarrow S^{2}$ must consist of a principal part which is a section, together with "bubbles" which lie in the fibers $M$ of $\pi: X_{b} \rightarrow S^{2}$; see McDuff [14, Lemma 2.9]. By assumption that $M$ is monotone, these bubbles must have positive Chern number. Using this, one can show that for a generic $\pi$-compatible family the evaluation map is a pseudocycle by standard arguments in McDuff and Salamon [17, Chapter 6].

\subsection{Definition of PGW invariants}

Under the assumptions of Proposition 2.14, we define parametric Gromov-Witten invariants by

$$
\mathrm{PGW}_{0, l}^{P}\left(a_{1}, \ldots, a_{l} ; A\right)=[\mathrm{ev}] \cdot\left(a_{1} \times \ldots \times a_{l}\right),
$$

where $\cdot$ denotes intersection pairing in $P^{l}$ and $a_{1}, \ldots, a_{l} \in H_{*}(P)$.

\subsection{Quantum homology}

The flavor of quantum homology we use is the following. Let $\Lambda:=\Lambda^{\text {univ }}\left[q, q^{-1}\right]$ be the ring of Laurent polynomials in a variable $q$ of degree 2 with coefficients in the universal Novikov ring. Thus, its elements are polynomials in $q$ of the form

$$
\sum_{\epsilon \in \mathbb{R}, \quad l \in \mathbb{Z}} \lambda_{\epsilon, l} \cdot q^{l} t^{\epsilon} \quad \#\left\{\lambda_{\epsilon, l} \neq 0 \mid \epsilon \geq c\right\}<\infty \text { for all } c \in \mathbb{R},
$$

where $\lambda_{\epsilon, l} \in \mathbb{Q}$. Set

$$
Q H_{*}(M)=Q H_{*}(M ; \Lambda)=H_{*}(M) \otimes_{\mathbb{Z}} \Lambda .
$$


We define a valuation $v: Q H_{*}(M) \rightarrow \mathbb{R}$ as follows:

$$
v\left(\sum_{A} b_{A} \cdot q^{l_{A}} t^{\epsilon_{A}}\right):=\sup _{b_{A} \neq 0} \epsilon_{A}
$$

where $A$ is an abstract index.

Recall that the quantum intersection product on $Q H_{*}(M)$ is defined as follows. For $a, b \in H_{*}(M)$

$$
a *_{M} b=\sum_{A \in H_{2}^{S}(M)}\left(a *_{M} b\right)_{A} \otimes q^{-c_{1}(A)} t^{-\omega(A)},
$$

where $\left(a *_{M} b\right)_{A} \in H_{i+j-2 n+2 c_{1}(A)}(M)$ is defined by the duality

$$
\left(a *_{M} b\right)_{A} \cdot c=G W_{0,3}^{M}(a, b, c ; A), \quad \text { for all } c \in H_{*}(M) .
$$

The product is then extended by linearity to all of $Q H_{*}(M)$. This product can be shown to be associative (see McDuff and Salamon [17, Chapter 11, Section 1] for details) and gives $Q H_{*}(M)$ the structure of a graded commutative ring with unit $[M]$.

\section{Definition of $\mathrm{QC}$ classes}

Let $X$ as before be a Hamiltonian fibration: $\pi: X \rightarrow S^{2}$ with monotone fiber $M$, and $p: P \rightarrow B$ be a smooth $\mathcal{F}$-fibration with fiber $X$, classified by a map into $L$ Ham, cf Remark 2.2. The following is a important ingredient in the definition of QC classes and plays the role of the 2 dimensional cohomology class of the curvature form in Chern-Weyl theory. Let $M_{\text {Ham }}$ denote the universal $M$ bundle over $B \operatorname{Ham}(M, \omega)$. There is a unique class $[\Omega] \in H^{2}\left(M_{\mathrm{Ham}}\right)$ called the coupling class such that

$$
i^{*}([\Omega])=[\omega], \quad \int_{M}[\Omega]^{n+1}=0 \in H^{2}(B \operatorname{Ham}(M, \omega))
$$

where $i: M \rightarrow M_{\mathrm{Ham}}$ is the inclusion of fiber, and the integral above denotes the integration along the fiber (see Kȩdra and McDuff [9, Section3]). Note from (8) that the total space $P$ of the bundle $p: P \rightarrow B$ has another submersive projection to $B \times S^{2}$ and the resulting bundle $M \hookrightarrow P \rightarrow B \times S^{2}$ is clearly Hamiltonian, ie the transition maps are fiberwise Hamiltonian symplectomorphisms.

Definition 3.1 We denote by $\mathcal{C} \in H^{2}(P)$, the pullback of the class [ $[\Omega]$ above, by the classifying map of the natural Hamiltonian fibration

$$
M \hookrightarrow P \rightarrow B \times S^{2} .
$$


Set $Q H_{*}^{B}(M)=H_{*}(B \times M) \otimes \Lambda$.

Definition 3.2 We define the total quantum characteristic class of $p: P \rightarrow B$ by

$$
c^{q}(P)=\sum_{A \in j_{*}\left(H_{2}^{\text {sect }}(X)\right)} b_{A} \otimes q^{-c_{\text {vert }}(A)} t^{-\mathcal{C}(A)} \in Q H_{*}^{B}(M) .
$$

In this formula,

- $H_{2}^{\text {sect }}(X)$ denotes the section homology classes of $\pi: X \rightarrow S^{2}$ as in Section 2.6.

- The map $j_{*}: H_{2}^{\text {sect }}(X) \rightarrow H_{2}\left(P_{f}\right)$ is induced by inclusion of fiber.

- The coefficient $b_{A} \in H_{*}(B \times M)$ is the transverse intersection of

$$
\text { ev }: \mathcal{M}_{0,1}\left(P, A ;\left\{J_{b}\right\}\right) \rightarrow P
$$

with $I_{0}(B \times M)$ (see (13)). More formally, $b_{A}$ is defined by duality

$$
b_{A} \cdot B \times M c=[\mathrm{ev}] \cdot P I_{0 *}(c),
$$

for $c \in H_{*}(B \times M)$.

The above definition works essentially without change for an $\mathcal{F}$-fibration classified by a map into $Q$.

Remark 3.3 To deduce that the condition (18) on the coefficients is satisfied we need to show that there are only finitely many homology classes $A \in H_{2}(P)$ which have representatives with area less then $c$ for every $c>0$ (for a fixed Riemannian metric on the compact manifold $P$ ). For then in particular there are only finitely many homology classes represented by vertical $\left\{J_{b}\right\}$-holomorphic curves with

$$
E(A)=\Omega_{b}(A)=\mathcal{C}(A)+\pi^{*}\left(\alpha_{b}\right)(A) \leq c
$$

for every $c>0$, which would imply the finiteness condition. To prove the former intuitive statement, one can use geometric measure theory and compactness theorem for spaces of integral currents with uniformly bounded mass norm (cf Federer [4]).

Notation 3.4 Let us from now on shorten notation by setting

$$
q^{-c_{\mathrm{vert}}(A)} t^{-\mathcal{C}(A)} \equiv e^{A},
$$

where it presents no confusion. 
For a regular family $\left\{J_{b}\right\}, \mathcal{M}_{0,1}^{*}\left(P, A,\left\{J_{b}\right\}\right)$ is a smooth manifold of dimension

$$
(2 n+2)+m+2 c_{1}(A)-4=(2 n+2)+m+2 c_{\mathrm{vert}}(A),
$$

where $m=\operatorname{dim} B(\operatorname{cf}(17))$. It follows that

$$
\operatorname{deg} b_{A}=2 n+m+2 c_{\text {vert }}(A) .
$$

In particular, a class $A$ contributes only if $2 c_{\text {vert }}(A) \leq 0$.

Every element $e=\sum_{j_{*}(A)} b_{A} \otimes e^{A} \in Q H_{*}^{B}(M)$ defines a linear functional on $H_{*}(B)$ (where $H_{k}(B)=H_{k}(B, \mathbb{Z}) /$ Tor) with values in $Q H_{*}(M)$ defined as follows. If $a \in H_{k}(B)$, then $e(a) \in Q H_{*}(M)$ is given by

$$
e(a)=\sum_{A} \sum_{i}\left(b_{A} \cdot\left(a \otimes e_{i}^{*}\right)\right) e_{i} \otimes e^{A},
$$

where $\left\{e_{i}\right\}$ is a basis for $H_{*}(M),\left\{e_{i}^{*}\right\}$ a dual basis for $H_{*}(M)$ with respect to the intersection pairing and $\cdot$ is the intersection pairing on $H_{*}(B \times M)$.

Remark 3.5 Let us check the degree of $e(a)$. We have that $b_{A} \cdot\left(a \otimes e_{i}^{*}\right)$ is nonzero when

$$
2 n+m+2 c_{\mathrm{vert}}(A)+\operatorname{deg} a+2 n-\operatorname{deg} e_{i}=2 n+m
$$

so we get $\operatorname{deg} e_{i}=2 n+\operatorname{deg} a+2 c_{\text {vert }}(A)$ and

$$
\operatorname{deg} e(a)=\operatorname{deg} e_{i}-2 c_{\mathrm{vert}}(A)=2 n+\operatorname{deg} a .
$$

Definition 3.6 We define the $k$-th quantum characteristic class

$$
c_{k}^{q}(P) \in \operatorname{Hom}\left(H_{k}(B), Q H_{*}(M)\right)=H^{k}\left(B, Q H_{*}(M)\right),
$$

to be the restriction of the functional $c^{q}(P)$ to $H_{k}(B)$.

In these terms, the functional $c^{q}(P)$ is just the sum

$$
c^{q}(P)=c_{0}^{q}(P)+c_{1}^{q}(P)+\ldots+c_{m}^{q}(P),
$$

where $m$ is the dimension of $B$. When $\gamma$ is contractible, Axiom 1 implies that $c_{0}^{q}(P)(\mathrm{pt})=[M]$ is the multiplicative identity in the quantum homology ring. The analogous expression for Chern classes is called the total Chern class. Interestingly, in our "quantum" setting the total class has a nice geometric interpretation and this plays a role in proving the corresponding "Whitney sum formula" in Section 4.4. 
Example 3.7 A loop $\gamma: S^{1} \rightarrow \operatorname{Ham}(M, \omega)$ can be viewed as a map $f_{\gamma}: \mathrm{pt} \rightarrow L$ Ham. The corresponding fibration $P_{f_{\gamma}}$ over pt has fiber $X_{\gamma}$ and

$$
c^{q}\left(P_{f_{\gamma}}\right)=\sum_{j_{*}(A)} b_{A} \otimes q^{-c_{\text {vert }}(A)} t^{-\widetilde{\omega}(A)} \in Q H_{2 n}(M),
$$

since $H_{*}(\mathrm{pt} \times M) \simeq H_{*}(M)$ and (21) implies that the degree of the element $c^{q}\left(P_{f_{\gamma}}\right)$ is $2 n$. In these terms, the Seidel element corresponding to $\gamma$ is defined to be

$$
S(\gamma)=c^{q}\left(P_{f_{\gamma}}\right) .
$$

This element depends only on the homotopy class of $\gamma$, and Seidel [24] proved that this defines a homomorphism $S: \pi_{1}(\operatorname{Ham}(M, \omega)) \rightarrow Q H_{2 n}(M)$.

Recall from the introduction that for a smooth $k$-cycle $f: B \rightarrow Q$,

$$
\Psi(B, f) \equiv c_{k}^{q}\left(P_{f}\right)([B]) .
$$

Lemma 3.8 The characteristic classes $c_{k}^{q}(P)$ of $p: P \rightarrow B$ are independent of the choice of the admissible family $\left\{\Omega_{b}\right\}$, and moreover $\Psi\left(B_{1}, f_{1}\right)=\Psi\left(B_{2}, f_{2}\right)$ if $f_{1}: B_{1} \rightarrow Q$ is oriented cobordant to $f_{2}: B_{2} \rightarrow Q$, in particular $\Psi$ is well defined map on $H_{*}(\Omega \operatorname{Ham}(M, \omega), \mathbb{Q})$.

Proof To prove that $c_{k}^{q}(P)$ are independent of the choice of the admissible family $\left\{\Omega_{b}\right\}$ note that by Lemma 2.5 any two such families are smoothly homotopy equivalent. The homotopy $\left\{\Omega_{b}^{t}\right\}$ gives an admissible family of forms on $p: P \times I \rightarrow I$ at which point we may apply Lemma 2.13 and Proposition 2.14.

To prove the second statement consider a smooth oriented cobordism $F: C \rightarrow Q$ between $\left(B_{1}, f_{1}\right)$ and $\left(B_{2}, f_{2}\right)$. The proof is just a simple consequence of Lemma 2.13. The construction in Section 2.1 yields an $\mathcal{F}$-fibration $P_{F}$ over $C$ restricting to the $\mathcal{F}$-fibrations $P_{i}$ over $B_{i}$. Moreover, for $c \in H_{*}(M)$ the class $I_{0}([C] \otimes c)$ in $P_{F}$ restricts to the corresponding classes $I_{0}\left(\left[B_{i}\right] \otimes c\right)$ in $P_{i}, \operatorname{cf}(13)$. Let

$$
\Psi\left(B_{i}, f_{i}\right)=\sum_{A} b_{A}^{i} \otimes e^{A}
$$

be the corresponding elements in $Q H_{*}(M)$. We need to show that $b_{A}^{1}=b_{A}^{2}$. Consider the intersection numbers

$$
b_{A}^{i} \cdot M^{c} c \equiv\left[\operatorname{ev}_{A}^{i}\right] \cdot P I_{0}\left(\left[B_{i}\right] \otimes c\right),
$$

where $\mathrm{ev}_{A}^{i}$ are the evaluation maps

$$
\mathrm{ev}_{A}^{i}: \mathcal{M}_{0,1}\left(P_{i}, A,\left\{J_{b}^{i}\right\}\right) \rightarrow P_{i}
$$


for regular families $\left\{J_{b}^{i}\right\}$. Let

$$
\mathrm{ev}_{A}^{F}: \mathcal{M}_{0,1}\left(P_{F}, A,\left\{J_{b}^{C}\right\}\right) \rightarrow P_{F}
$$

be the evaluation map with $\left\{J_{b}^{C}\right\}$ a regular family restricting to $\left\{J_{b}^{i}\right\}$ on $P_{i}$. When the dimension of $c$ is such that the intersection numbers above are nonzero, Lemma 2.13, and Proposition 2.14 imply that $\operatorname{ev}_{A}^{F} \cap I([C] \otimes c)$ is a one-dimensional cobordism between the oriented 0-dimensional manifolds $\operatorname{ev}_{A}^{1} \cap I_{0}\left(B_{1} \otimes c\right), \mathrm{ev}_{A}^{2} \cap I_{0}\left(B_{2} \otimes c\right)$, assuming things are perturbed to be transverse. Thus, the intersection numbers $\mathrm{ev}_{A}^{1} \cdot I_{0}\left(B_{1} \otimes c\right), \mathrm{ev}_{A}^{2} \cdot I_{0}\left(B_{2} \otimes c\right)$ coincide.

To conclude that $\Psi$ is well defined on $H_{*}(\Omega \operatorname{Ham}(M, \omega), \mathbb{Q})$ we may use Theorem 6.1 , which implies that the rational homology of $\Omega \operatorname{Ham}(M, \omega)$ is generated by cycles $f: B \rightarrow X$, where $B$ is a closed oriented smooth $k$-manifold (in fact a product of spheres). Moreover, Theorem 6.1 implies that the relations in the rational homology of $\Omega \operatorname{Ham}(M, \omega)$ are generated by maps of smooth cobordisms (actually cylindrical cobordisms).

\section{Verification of axioms}

Proposition 4.1 Let $p: P \rightarrow B$ be an $\mathcal{F}$-fibration and $f: C \rightarrow B$ a smooth $k$-cycle representing $a \in H_{*}(B)$. Then

$$
c_{k}^{q}(P)(a)=\Psi\left(f^{*} P\right) \equiv c_{k}^{q}\left(f^{*} P([C])\right) .
$$

Proof Let $\left\{J_{b}\right\}$ be a regular family for $A$ curves in $P$. We have maps

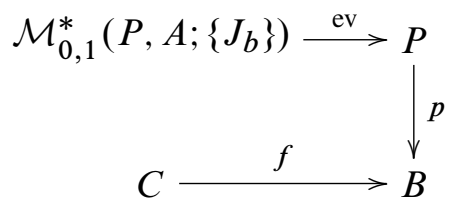

Perturb $f: C \rightarrow B$ to be transverse to the pseudocycle

$$
p \circ \mathrm{ev}: \mathcal{M}_{0,1}^{*}\left(P, A,\left\{J_{b}\right\}\right) \rightarrow B,
$$


and consider the commutative diagram

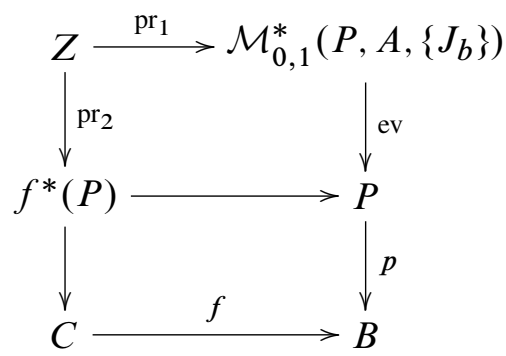

where $Z$ is the pullback of the diagram. By the transversality above $Z$ is a smooth manifold and can be tautologically identified with $\mathcal{M}_{0,1}^{*}\left(P^{\prime}, A,\left\{J_{b}^{\prime}\right\}\right)$, where $p^{\prime}: P^{\prime} \rightarrow$ $C$ is the pullback bundle $f^{*}(P)$ over $C$ and $\left\{J_{b}^{\prime}\right\}=\left\{f^{*}\left(J_{b}\right)\right\}$. Moreover, the evaluation map ev': $\mathcal{M}_{0,1}^{*}\left(P^{\prime}, A,\left\{J_{b}^{\prime}\right\}\right) \rightarrow P^{\prime}$ is just the map $\mathrm{pr}_{2}$ and is a pseudocycle since ev is a pseudocycle. The dimension of this pseudocycle is

$$
\operatorname{dim}[\mathrm{ev}]+k-m=\left(2 n+m+2 c_{1}(A)-2\right)+k-m=2 n+k+2 c_{1}(A)-2,
$$

where $m$ is the dimension of $B$ and the expected dimension of $\mathcal{M}_{0,1}^{*}\left(P^{\prime}, A,\left\{J_{b}^{\prime}\right\}\right)$. Thus, ev: $\mathcal{M}_{0,1}^{*}\left(P^{\prime}, A,\left\{J_{b}^{\prime}\right\}\right) \rightarrow P^{\prime}$ is a pseudocycle of the correct dimension.

We show that the family $\left\{J_{b}^{\prime}\right\}$ is regular. The linearized Cauchy-Riemann operator for $b$ in the intersection of $p \circ$ ev with $f$ has the form:

$$
D_{u, b}: \Omega_{B}^{0} \equiv\left\{\xi \in \Omega^{0}\left(S^{2}, u^{*} T P\right) \mid p_{*}(\xi) \equiv \mathrm{const}\right\} \rightarrow \Omega^{0,1}\left(S^{2}, u^{*} T X_{b}\right) .
$$

By the regularity assumption on $\left\{J_{b}\right\}$ this operator is onto. Moreover, by regularity we have

$$
p_{*}\left(\operatorname{ker} D_{u, b}\right)=p_{*} \circ \mathrm{ev}_{*}\left(T_{u} \mathcal{M}_{0,1}\left(P, J_{b}\right)\right) .
$$

Thus, by the transversality assumption we must have that

$$
p_{*}: \operatorname{ker} D_{u, b} \rightarrow T_{b} B /\left.f_{*}(T C)\right|_{b}
$$

is onto. Denote by $D_{u, b}^{C}$ the restriction of the operator $D_{u, b}$ to the subspace

$$
\Omega_{C}^{0} \equiv\left\{\xi \in \Omega^{0}\left(S^{2}, u^{*} T P \mid p_{*}(\xi) \equiv \text { const }\left.\in f_{*}(T C)\right|_{b}\right)\right\} .
$$

To show that $\left\{J_{b}^{\prime}\right\}$ is regular we must show that $D_{u, b}^{C}$ is also onto. Let $\widetilde{v} \in \Omega_{B}^{0}$. By (22) there exists $\widetilde{v}_{k} \in \operatorname{ker} D_{u, b}$ and $\left.v_{C} \in f_{*}(T C)\right|_{b}$, s.t.

$$
p_{*}(\widetilde{v})=p_{*}\left(\widetilde{v}_{k}\right)+v_{C} .
$$

Therefore, we get that $\tilde{v}-\widetilde{v}_{k} \in \Omega_{C}^{0}$, and so $D_{u, b}(\widetilde{v})=D_{u, b}^{C}\left(\tilde{v}-\widetilde{v}_{k}\right)$. Since $D_{u, b}$ is onto, it follows that $D_{u, b}^{C}$ is also onto and so $\left\{J_{b}^{\prime}\right\}$ is regular. 
By definition,

$$
\begin{gathered}
c_{k}^{q}(P)(a)=\sum_{A} \sum_{i} b_{A} \cdot\left(a \otimes e_{i}^{*}\right) e_{i} \otimes e^{A}, \\
c_{k}^{q}\left(P^{\prime}\right)([C])=\sum_{A} \sum_{i} b_{A}^{\prime} \cdot\left([C] \otimes e_{i}^{*}\right) e_{i} \otimes e^{A} .
\end{gathered}
$$

To finish the proof, we note that by the above discussion

$$
\begin{aligned}
& b_{A} \cdot B \times M\left(a \otimes e_{i}\right) \equiv[\mathrm{ev}] \cdot P I_{0 *}\left(a \otimes e_{i}\right) \\
& =\left[\mathrm{ev}^{\prime}\right] \cdot P^{\prime} I_{0 *}\left([C] \otimes e_{i}\right) \\
& \equiv\left[b_{A}^{\prime}\right] \cdot C \times M\left([C] \otimes e_{i}\right) \text {. }
\end{aligned}
$$

\subsection{Verification of Axiom 1}

To prove the first statement just apply Proposition 4.1 to $i: \mathrm{pt} \rightarrow B$. To prove the second statement note that if $P \simeq X \times B$ then we can take a constant family of regular compatible almost complex structures $\left\{J^{\text {reg }}\right\}$ and this family is clearly parametrically regular. It follows that the total characteristic class is

$$
c^{q}(P)=\sum_{A}\left(B \otimes b_{A}^{\prime}\right) \otimes e^{A},
$$

where $b_{A}^{\prime}$ is the transverse intersection of ev: $\mathcal{M}_{0,1}\left(X, A, J^{r e g}\right) \rightarrow X$ with the fiber $M_{0} \subset X$ over 0 . As a functional on $H_{*}(B), c^{q}(P)(a)=0$ unless $\operatorname{deg}(a)=0$.

\subsection{Verification of Axiom 2}

If $f: C \rightarrow B$ represents $a \in H_{k}\left(B_{1}\right)$ as before, then

$$
g^{*} c_{k}^{q}\left(P_{2}\right)\left(f_{*}[C]\right)=c_{k}^{q}\left(P_{2}\right)\left(g_{*} f_{*}[C]\right)=\Psi\left(f^{*} g^{*} P_{2}\right),
$$

where the last equality holds by Proposition 4.1, and

$$
c_{k}^{q}\left(g^{*} P_{2}\right)\left(f_{*}[C]\right)=\Psi\left(f^{*} g^{*} P_{2}\right),
$$

again by Proposition 4.1.

\subsection{Proof of Theorem 1.9 assuming Axiom 3}

Definition 4.2 The Pontryagin product

$$
f_{1} \star f_{2}: B_{1} \times B_{2} \rightarrow \Omega \operatorname{Ham}(M, \omega)
$$


of two maps $f_{1}, f_{2}: B_{1}, B_{2} \rightarrow \Omega \operatorname{Ham}(M, \omega)$ is defined by

$$
f_{1} \star f_{2}\left(b_{1}, b_{2}, \theta\right)=f_{2}\left(b_{2}, \theta\right) \circ f_{1}\left(b_{1}, \theta\right) .
$$

(The order is indeed reversed.)

Proof For $i=1,2$, let $f_{i}: B_{i} \rightarrow L$ Ham be as before. Let $k_{i}$ be the dimension of $B_{i}$. Consider the maps

$$
\tilde{f}_{i}: B_{1} \times B_{2} \rightarrow \Omega \operatorname{Ham}(M, \omega), \quad \tilde{f}_{i}=f_{i} \circ \mathrm{pr}_{i} \quad \text { for } i=1,2,
$$

where $\mathrm{pr}_{i}: B_{1} \times B_{2} \rightarrow B_{i}$ are the component projections. Clearly,

$$
P_{f_{1} \star f_{2}} \simeq P_{\widetilde{f}_{1}} \oplus P_{\tilde{f}_{2}} \equiv P \widetilde{f}_{2} \cdot \widetilde{f}_{1}
$$

(see Definition 1.4). By Axiom 2 and Axiom 3,

$$
\begin{aligned}
\Psi\left(B_{1} \times B_{2}, f_{1} \star f_{2}\right) & \equiv c_{k_{1}+k_{2}}^{q}\left(P_{f_{1} \star f_{2}}\right)\left(B_{1} \times B_{2}\right) \\
& =\sum_{i+j=k_{1}+k_{2}} \operatorname{pr}_{1}^{*}\left(c_{i}^{q}\left(P_{f_{1}}\right)\right) \cup \operatorname{pr}_{2}^{*}\left(c_{j}^{q}\left(P_{f_{2}}\right)\right)\left(B_{1} \times B_{2}\right) \\
& =\operatorname{pr}_{1}^{*}\left(c_{k_{1}}^{q}\left(P_{f_{1}}\right)\right) \cup \operatorname{pr}_{2}^{*}\left(c_{k_{2}}^{q}\left(P_{f_{2}}\right)\right)\left(B_{1} \times B_{2}\right) \\
& =c_{k_{1}}^{q}\left(P_{f_{1}}\right)\left(B_{1}\right) * c_{k_{2}}^{q}\left(P_{f_{2}}\right)\left(B_{2}\right) \\
& =\Psi\left(B_{1}, f_{1}\right) * \Psi\left(B_{2}, f_{2}\right) .
\end{aligned}
$$

Remark 4.3 Under the Pontryagin product, the group ring of $\pi_{1}(\operatorname{Ham}(M, \omega))$ over $\mathbb{Q}$ is $H_{0}(\Omega \operatorname{Ham}(M, \omega), \mathbb{Q})$. The restriction of $\Psi$ to degree zero,

$$
\Psi^{0}: H_{0}(\Omega \operatorname{Ham}(M, \omega), \mathbb{Q}) \rightarrow Q H_{2 n}(M),
$$

is a ring homomorphism

$$
S=\Psi^{0}: \mathbb{Q}\left[\pi_{1}(\operatorname{Ham}(M, \omega))\right] \rightarrow Q H_{2 n}(M),
$$

in view of (2). Thus, Theorem 1.9 is an extension of the Seidel homomorphism $S$ to the entire Pontryagin ring $H_{*}(\Omega \operatorname{Ham}(M, \omega), \mathbb{Q})$.

\subsection{Verification of Axiom 3}

In this subsection we prove that the classes $c_{k}^{q}$ satisfy Axiom 3. To this end we will need a splitting formula for PGW invariants arising from the connected sum operation on two $\mathcal{F}$-fibrations. To help clarify the picture we first explain why $P_{1} \oplus P_{2}$ is the connected sum of $P_{1}, P_{2}$ in an appropriate way. 
Definition 4.4 Let $P_{1}, P_{2}$ be two $\mathcal{F}$-fibrations classified by $f^{1}, f^{2}: B \rightarrow L$ Ham. Define

$$
P_{1} \# P_{2} \equiv\left(B \times M \times D_{0}^{2}\right) \cup\left(B \times M \times S^{1} \times I\right) \cup\left(B \times M \times D_{\infty}^{2}\right) / \sim
$$

where the equivalence relation is

$$
\begin{aligned}
(b, x, 1, \theta)_{0} & \sim\left(b, f_{b, \theta}^{1}(x), \theta, 0\right) \in B \times M \times S^{1} \times I \\
(b, x, 1, \theta)_{\infty} & \sim\left(b,\left(f_{b, \theta}^{2}\right)^{-1}(x), \theta, 1\right) \in B \times M \times S^{1} \times I .
\end{aligned}
$$

It is then not hard to construct a natural isomorphism between $P_{1} \oplus P_{2}$ and $P_{1} \# P_{2}$. Given classes $A$ and $B$ in $j_{*} H_{2}^{\text {sect }}\left(X_{1}\right) \subset H_{2}\left(P_{1}\right)$ respectively $j_{*} H_{2}^{\text {sect }}\left(X_{2}\right) \subset H_{2}\left(P_{2}\right)$, there is a natural section class $A \# B$ in $H_{2}\left(P_{1} \# P_{2}\right)$. To define this class one represents $A$ and $B$ by sections coinciding in the fiber over $\infty$ for $X_{b}^{1}$ and the fiber over 0 for $X_{b}^{2}$ respectively, (this can be made more precise using the definition above). It can be directly checked that the class $A \# B$ is independent of choices and satisfies

$$
c_{\text {vert }}(A \# B)=c_{\text {vert }}(A)+c_{\text {vert }}(B) \text { and } \mathcal{C}_{P_{1} \# P_{2}}(A \# B)=\mathcal{C}_{P_{1}}(A)+\mathcal{C}_{P_{2}}(B) .
$$

Given $a \in H_{*}(B \times M)$, we will denote the class $\left(I_{z}^{P}\right)_{*}(a) \in H_{*}(P)$ by $a$ for shorthand and similarly for $P_{1}, P_{2}$.

Theorem 4.5 Let $P_{1}, P_{2}$ be two $\mathcal{F}$-fibrations classified by $f^{1}, f^{2}: B \rightarrow$ LHam, $P=P_{1} \oplus P_{2}$ their connected sum, $A \in H_{2}\left(P_{1}\right), B \in H_{2}\left(P_{2}\right)$ as in Definition 1.5. Then for all $a_{1}, \ldots, a_{k} \in H_{*}(B \times M)$, and any integer $0 \leq l \leq k$,

$$
\begin{array}{r}
\mathrm{PGW}_{0, k}^{P}\left(a_{1}, \ldots, a_{k} ; C\right) \\
=\sum_{\substack{i \\
A_{1} \# B_{1}=C}} \operatorname{PGW}_{0, l+1}^{P_{2}}\left(a_{1}, \ldots, a_{l}, e_{i} ; A_{1}\right) \\
\cdot \operatorname{PGW}_{0, k-l+1}^{P_{1}}\left(e_{i}^{*}, a_{l+1}, \ldots, a_{k} ; A_{2}\right),
\end{array}
$$

where $\left\{e_{i}\right\}$ is a basis for $H_{*}(B \times M),\left\{e_{i}^{*}\right\}$ is the dual basis.

Proof Set $K=B \times M$. Suppose we have two $J$-holomorphic curves $u_{1}, u_{2}$ into $P_{f^{1}}$ and $P_{f^{2}}$ in class $A_{1}, A_{2}$ intersecting in $K$, (where we identify $K$ with its embedding in $P_{f}{ }^{1}$ by $I_{\infty}$ and in $P_{f}{ }^{2}$ by $I_{0}$ ), then $u_{1}, u_{2}$ lie in the respective fibers $X_{b}^{1}$ and $X_{b}^{2}$ over the same point $b \in B$. We can then glue them to get a curve in the fiber $X_{b}^{1} \# X_{b}^{2} \simeq X_{f^{2}(b) \cdot f^{1}(b)}$ over $b$ of the fibration $P_{1} \oplus P_{2}$ by exactly same argument as in Section 11.4 of [17].

One then shows that for generic families $\left\{J_{b}^{1}\right\},\left\{J_{b}^{2}\right\}$ the moduli spaces

$$
\mathcal{M}^{*}\left(P_{f^{1}}, A_{1} ;\left\{J_{b}^{1}\right\}\right) \text { and } \mathcal{M}^{*}\left(P_{f^{2}}, A_{2} ;\left\{J_{b}^{2}\right\}\right)
$$


are regular and the evaluation map

$$
\mathcal{M}^{*}\left(P_{f^{1}}, A_{1} ;\left\{J_{b}^{1}\right\}\right) \times \mathcal{M}^{*}\left(P_{f^{2}}, A_{2} ;\left\{J_{b}^{2}\right\}\right) \rightarrow K \times K
$$

which takes $\left(u^{1}, u^{2}\right) \mapsto\left(u_{\infty}^{1}(0), u_{0}^{2}(0)\right)$ is transverse to the diagonal. The rest of the proof is exactly the same as the proof of the corresponding splitting statement in Chapter 10 of [17].

Remark 4.6 Note that since all holomorphic curves of $P_{1}, P_{2}, P_{1} \# P_{2}$ come from section classes (of the fiber $X^{1}, X^{2}, X^{1} \# X^{2}$ respectively) they are necessarily transverse to the divisor $K$ and intersect it in a single point. This formula is then "essentially" a special case of the formula given by Ionel and Parker [8] for general symplectic sums along a codimension 2 submanifold; see also Li and Ruan [12] for a different approach. The main difference in our case is that we don't have global symplectic forms on $P_{1}, P_{2}, P_{1} \# P_{2}$ but rather families of forms.

In what follows we think of $H^{*}\left(B, Q H_{*}(M)\right)$ as the space of linear functionals on $H_{*}(B)$ with values in $Q H_{*}(M)$. In particular an element in $H^{*}\left(B, Q H_{*}(M)\right)$ can be of mixed degree. Thus, by the Kunneth formula and Poincare duality $H^{*}\left(B, Q H_{*}(M)\right)$ is naturally identified with $Q H_{*}^{B}(M)$ via (20). To avoid confusion for an element $a \in$ $Q H_{*}^{B}(M)$ we will write $\operatorname{PD}(a)$ when we think of it as an element of $H^{*}\left(B, Q H_{*}(M)\right)$.

Considering the following elements $a, b \in H_{*}(B \times M) \subset Q H_{*}^{B}(M)$

$$
a=\sum_{i} a_{i} \otimes m_{i}, \quad a^{\prime}=\sum_{j} a_{j}^{\prime} \otimes m_{j}^{\prime}, \quad a_{i}, a_{i}^{\prime} \in H_{*}(B), m_{i}, m_{j}^{\prime} \in H_{*}(M),
$$

then

$$
\mathrm{PD}(a) \cup \operatorname{PD}\left(a^{\prime}\right)=\operatorname{PD}\left(\sum_{i, j}\left(a_{i} \cap a_{j}^{\prime}\right) \otimes m_{i} * m_{j}^{\prime}\right),
$$

where $*$ is the homology quantum product. We will need the following simple Lemma.

Lemma 4.7 If $P_{\mathrm{tr}}=B \times\left(M \times S^{2}\right)$ and $a, b$ as above, then

$$
\begin{aligned}
\mathrm{PD}(a) \cup \operatorname{PD}(b) & =\operatorname{PD}\left(\sum_{A}(\mathrm{PD}(a) \cup \operatorname{PD}(b))_{A} e^{A} \in Q H_{*}^{B}(M)\right), \\
(\mathrm{PD}(a) \cup \operatorname{PD}(b))_{A} & =\sum_{k, l} \operatorname{PGW}_{0,3}^{P_{\mathrm{tr}}}\left(a, a^{\prime}, e_{k, l} ; A\right) e_{k, l}^{*},
\end{aligned}
$$

where $\left\{e_{k, l}=b_{k} \otimes e_{l}\right\}$ is a basis for $H_{*}(B \times M)$. 
Proof Let $\left\{J^{\text {reg }}\right\}$ be the constant family of regular complex structures on $P_{\text {tr }}$ compatible with a constant admissible family $\{\Omega\}$. Then the family $\left\{J^{\text {reg }}\right\}$ is itself parametrically regular. We have

$$
\sum_{k, l} \mathrm{PGW}_{0,3}^{P_{\mathrm{tr}}}\left(a, b, e_{k, l} ; A\right) e_{k, l}^{*}=\sum_{k, l} \sum_{i, j} \mathrm{PGW}_{0,3}^{P_{\mathrm{tr}}}\left(a_{i} \otimes m_{i}, a_{j}^{\prime} \otimes m_{j}^{\prime}, e_{k, l} ; A\right) e_{k, l}^{*} .
$$

As oriented manifolds,

$$
\mathcal{M}_{0,3}^{*}\left(P_{\mathrm{tr}}, A ; J^{\mathrm{reg}}\right) \simeq B \times \mathcal{M}_{0,3}^{*}\left(\operatorname{tr}, A ;\left\{J^{\mathrm{reg}}\right\}\right) .
$$

Moreover the diagram

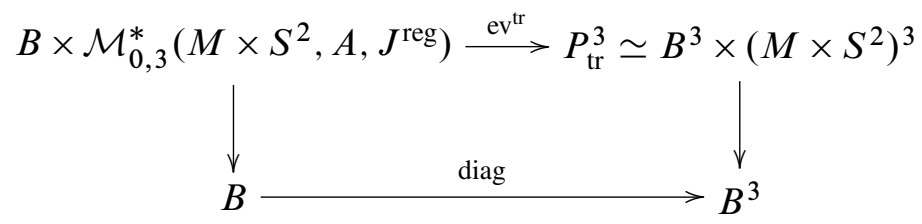

commutes. Hence, $\left[\mathrm{ev}^{P_{\mathrm{tr}}}\right]=[\mathrm{diag}] \otimes\left[\mathrm{ev}^{M \times S^{2}}\right]$ as a cycle in $B^{3} \times\left(M \times S^{2}\right)^{3}$ with the orientation pulled back from the orientation on $\operatorname{tr}^{3}$ via the identification $\operatorname{tr}^{3} \simeq B^{3} \times\left(M \times S^{2}\right)^{3}$, where $\mathrm{ev}^{\mathrm{tr}}$ and $\mathrm{ev}^{M \times S^{2}}$ are the evaluation maps from $\mathcal{M}_{0,3}^{*}\left(\operatorname{tr}, A,\left\{J^{\text {reg }}\right\}\right)$ and $\mathcal{M}_{0,3}^{*}\left(M \times S^{2}, A, J^{\text {reg }}\right)$, respectively.

Therefore,

$$
\begin{aligned}
\sum_{k, l} \operatorname{PGW}_{0,3}^{\mathrm{Ttr}} & \left(a_{i} \otimes m_{i}, a_{j}^{\prime} \otimes m_{j}^{\prime}, e_{k, l} ; A\right) e_{k, l}^{*} \\
& =\sum_{k, l}\left(\left[\mathrm{ev}^{\mathrm{tr}}\right] \cdot\left(a_{i} \otimes a_{j}^{\prime} \otimes b_{k}\right) \otimes\left(m_{i} \otimes m_{j}^{\prime} \otimes e_{l}\right)\right)\left(b_{k} \otimes e_{l}\right)^{*} \\
& =\sum_{k, l}[\mathrm{diag}] \otimes\left[\mathrm{ev}^{M \times S^{2}}\right] \cdot\left(a_{i} \otimes a_{j}^{\prime} \otimes b_{k}\right) \otimes\left(m_{i} \otimes m_{j}^{\prime} \otimes e_{l}\right)\left(b_{k} \otimes e_{l}\right)^{*} \\
& =\sum_{k}\left(\left(a_{i} \cap a_{j}^{\prime}\right) \cdot b_{k}\right) b_{k}^{*} \otimes \sum_{l} G W_{0,3}^{M \times S^{2}}\left(m_{i}, m_{j}, e_{l} ; A\right) e_{l}^{*} \\
& =\left(a_{i} \cap a_{j}^{\prime}\right) \otimes \sum_{l} G W_{0,3}^{M \times S^{2}}\left(m_{i}, m_{j}, e_{l} ; A\right) e_{l}^{*},
\end{aligned}
$$

where we used that $[\mathrm{diag}] \cdot a_{i} \otimes a_{j}^{\prime} \otimes b_{k}=\left(a_{i} \cap a_{j}^{\prime}\right) \cdot b_{k}$. Summing over all $A \in$ $H_{2}^{\text {sect }}\left(M \times S^{2}\right)$ we get the desired equality.

Given an $\mathcal{F}$-fibration $P$, define $m^{P}: H_{*}(B \times M) \rightarrow Q H_{*}^{B}(M)$ by

$$
m^{P}(a)=\sum_{A, i} \operatorname{PGW}_{0,2}^{P}\left(a, e_{i} ; A\right) e_{i}^{*} \otimes e^{A},
$$


where $e_{i}$ are as in Theorem 4.5, and extend by linearity to all of $Q H_{*}^{B}(M)$.

Lemma 4.8 If $P=P_{1} \oplus P_{2}$ then

$$
m^{P}=m^{P_{1}} \circ m^{P_{2}} .
$$

Proof By (23),

$$
\begin{aligned}
m^{P_{1}} \circ m^{P_{2}}(a) & =\sum_{C} \sum_{i, A \# B=C} \operatorname{PGW}_{0,2}^{P_{2}}\left(a, e_{i} ; A\right) \cdot \mathrm{PGW}_{0,2}^{P_{1}}\left(e_{i}^{*}, e_{j} ; B\right) e_{j}^{*} \otimes e^{C} \\
& =\sum_{i, C} \operatorname{PGW}_{0,2}^{P}\left(a, e_{j}, C\right) e_{j}^{*} \otimes e^{C}=m^{P}(a),
\end{aligned}
$$

where we used Theorem 4.5 for the second equality.

Lemma 4.9 For an $\mathcal{F}$-fibration $P$,

$$
\mathrm{PD}\left(m^{P}(a)\right)=\operatorname{PD}\left(c^{q}(P)\right) \cup \operatorname{PD}(a) .
$$

Proof It suffices to prove this for a simple class $a \in Q H_{*}^{B}(M)$. Using Theorem 4.5 with $P_{2}=P$ and $P_{1}=\operatorname{tr}=B \times\left(M \times S^{2}\right)$ we get:

$$
\begin{aligned}
\operatorname{PD}\left(m^{P}(a)\right) & =\operatorname{PD}\left(\sum_{j, C} \operatorname{PGW}_{0,2}^{P}\left(a, e_{j} ; C\right) e_{j}^{*} \otimes e^{C}\right) \\
& =\operatorname{PD}\left(\sum_{i, j, A \# B=C} \operatorname{PGW}_{0,1}^{P}\left(e_{i} ; A\right) \cdot \mathrm{PGW}_{0,3}^{P_{\mathrm{tr}}}\left(e_{i}^{*}, a, e_{j} ; B\right) e_{j}^{*} \otimes e^{C}\right) \\
& =\sum_{i, A \# B=C} \operatorname{PGW}_{0,1}^{P}\left(e_{i} ; A\right) \cdot\left(\operatorname{PD}\left(e_{i}^{*}\right) \cup \operatorname{PD}(a)\right)_{B} \otimes e^{C} \\
& =\operatorname{PD}\left(c^{q}(P)\right) \cup \operatorname{PD}(a),
\end{aligned}
$$

where we used Lemma 4.7 for the next to last equality.

\subsection{Verification of Axiom 3}

Using the above lemmas we get,

$$
\begin{aligned}
\mathrm{PD}\left(c^{q}(P)\right)=\mathrm{PD}\left(m^{P}(B \times M)\right) & =\mathrm{PD}\left(m^{P_{1}} \circ m^{P_{2}}(B \times M)\right) \\
& =\mathrm{PD}\left(c^{q}\left(P_{1}\right)\right) \cup \operatorname{PD}\left(c^{q}\left(P_{2}\right)\right) .
\end{aligned}
$$




\section{QC classes and the Hofer geometry}

Let $p: P_{f} \rightarrow B$ be a smooth $\mathcal{F}$-fibration. We explain here how $c^{q}\left(P_{f}\right)$ gives rise to lower bounds for the positive max-length measure $L^{+}(f)=\max _{b \in B} L^{+}\left(f_{b}\right)$; this will be used later in this section. We will assume that the family $\left\{\Omega_{b}\right\}$ on $P_{f}$ has been chosen so that condition (9) is satisfied. Let $f: B \rightarrow Q$ be a general smooth cycle. Define a valuation

$$
\nu: Q H_{*}(M), Q H_{*}^{B}(M) \rightarrow \mathbb{R} \quad \text { by } \quad v\left(\sum_{A} b_{A} \cdot t^{\epsilon_{A}} q^{l_{A}}\right):=\sup _{b_{A} \neq 0} \epsilon_{A},
$$

and $b_{A}$ is in $H_{*}(M)$ or $H_{*}(B \times M)$. Our next proposition is a direct generalization of Seidel's (see McDuff [15]).

Proposition 5.1 We have

$$
v\left(c^{q}\left(P_{f}\right)\right) \leq \min _{(B, f) \in[H]}\left(\max _{b \in B} L^{+}\left(\gamma_{b}\right)\right),
$$

where $[H]$ represents the homotopy class of maps $f: B \rightarrow Q$ and $\gamma_{b}$ is the loop $f(b)$ (this is defined up to an action of $S^{1}$ ). Moreover,

$$
v(\Psi(B, f)) \leq \min _{(B, f) \in[H]}\left(\max _{b \in B} L^{+}\left(\gamma_{b}\right)\right),
$$

where $[H]$ now denotes the bordism class of maps $f: B \rightarrow Q$.

\section{Proof Let}

$$
c^{q}(P)=\sum_{A} b_{A} \otimes q^{-c_{\mathrm{vert}}(A)} t^{-\mathcal{C}(A)} .
$$

If $b_{A} \neq 0$ in $H_{*}(B \times M)$ then there is a $J_{b}$-holomorphic curve $u:\left(S^{2}, j\right) \rightarrow X_{b} \subset P_{f}$ in class $A \in H_{2}^{S}\left(P_{f}\right)$. On the other hand $\left[\Omega_{b}\right]=\mathcal{C}+\pi^{*}\left(\left[\alpha_{b}\right]\right)$, for some area form $\alpha$ on $S^{2}$, where $\left[\Omega_{b}\right]$ is the cohomology class of $\Omega_{b}$ in $H^{2}\left(X_{b}\right)$. Since $\Omega_{b}$ tames $J_{b}$, we get

$$
0<\left[\Omega_{b}\right](A)=\left(\mathcal{C}+\pi^{*}\left(\alpha_{b}\right)\right)(A)=\mathcal{C}(A)+\operatorname{area}\left(p^{-1}(b), \Omega_{b}\right) .
$$

Therefore,

$$
-\mathcal{C}(A)<\operatorname{area}\left(p^{-1}(b), \Omega_{b}\right)=L^{+}\left(\gamma_{b}\right)+2 \epsilon \leq \max _{b \in B} L^{+}\left(\gamma_{b}\right)+2 \epsilon
$$

for all $A$. Passing to the limit in $A$ and $\epsilon$ we get $v\left(c^{q}\left(P_{f}\right)\right) \leq \max _{b \in B} L^{+}\left(\gamma_{b}\right)$. Since the left hand side of (26) depends only on the homotopy class of $f$, we get the inequality (24). Inequality (25) follows by the same argument and Lemma 3.8. 


\subsection{Calculation for some symmetric $\mathcal{F}$-fibrations}

Let $q: Y \rightarrow B$ be a principal $S^{1}$-bundle and $\hat{f}: Y \rightarrow \operatorname{Ham}(M, \omega)$ an $S^{1}$-equivariant map with respect to the right action of some subgroup $\gamma: S^{1} \rightarrow \operatorname{Ham}(M, \omega)$ on $\operatorname{Ham}(M, \omega)$. Recall from Section 2.5 that we have an induced cycle $f: B \rightarrow Q$, and an induced fibration $p: P_{f} \rightarrow B$. In this section, we give an expression for the "leading-order term" contribution to the total quantum characteristic class $c^{q}\left(P_{f}\right)$ and give a proof of Theorem 1.10. This extends the calculation for $S^{1}$ actions in [19, Theorem 1.10].

By Lemma 2.10, $P_{f}$ can be identified with $h: Y \times_{S^{1}} X_{\gamma} \rightarrow B$. The bundle $Y \times_{S^{1}} X_{\gamma}$ comes with an admissible family $\left\{\Omega_{b}\right\}$ and a compatible family $\left\{J_{b}\right\}$ constructed in Section 2.5.1. To understand the behavior of fiber holomorphic curves in $P_{f}$, we need to first understand $\widetilde{J}$ holomorphic curves in $X_{\gamma}$, where $\widetilde{J}$ is the almost complex structure described in Section 2.5.1. Each fixed point $x$ of the $S^{1}$-action $\gamma$ gives rise to a $\widetilde{J}$-holomorphic section of $X_{\gamma}$ defined by

$$
\sigma_{x}=S^{3} \times_{S^{1}}\{x\} \subset X_{\gamma} .
$$

Denote by $F_{\max }$ the maximal fixed point set of the Hamiltonian $S^{1}$-action $\gamma$ on $M$, ie the maximal set of the generating Hamiltonian $H$ of $\gamma$. Let $\sigma_{\max } \in H_{2}\left(X_{\gamma}\right)$ denote the homology class of the section $\sigma_{x}$ for $x \in F_{\max }$. For each $x \in F_{\max }$ we have a $\widetilde{J}$ holomorphic $\sigma_{\max }$-class curve. An important observation due to Seidel is that these are the only $\widetilde{J}$-holomorphic curves in that homology class (cf [19, Lemma 3.1]); and so the moduli space of these unparametrized curves is identified with $F_{\max }$. Since the $S^{1}$-action $\beta$ (see (14)) on $X_{\gamma}$ maps each section $\sigma_{x}$ to itself, it follows that the unparametrized moduli space $\mathcal{M}_{0,0}^{*}\left(P_{f}, \sigma_{\max } ;\left\{J_{b}\right\}\right)$ can be identified with $B \times F_{\max }$. In particular it is a compact manifold. Let $E$ be the obstruction bundle over this moduli space. The fiber of $E$ at $(b, x) \in B \times F_{\max }$ is the cokernel of the operator

$$
D_{u, b}:\left\{\xi \in \Omega^{0}\left(S^{2}, u^{*} T P_{f}\right) \mid d p_{f}(\xi) \equiv \text { const }\right\} \rightarrow \Omega^{0,1}\left(S^{2}, u^{*} T X_{b}\right),
$$

where $u$ : $S^{2} \rightarrow X_{b}$ parametrizes the section $\sigma_{x} \subset X_{b}$. We write $D_{u, b}^{\text {vert }}$ for the restriction of $D_{u, b}$ to $\Omega^{0}\left(S^{2}, u^{*} T X_{b}\right)$.

Lemma $5.2 \operatorname{coker} D_{u, b}^{\mathrm{vert}} \simeq \operatorname{coker} D_{u, b}$.

Proof Since the map

$$
p_{f} \circ \mathrm{ev}: \mathcal{M}_{0,1}^{*}\left(P_{f}, \sigma_{\max } ;\left\{J_{b}\right\}\right) \rightarrow B
$$

is a submersion, the homomorphism

$$
d p_{f}: \operatorname{ker} D_{u, b} \rightarrow T_{b} B
$$


is onto. It easily follows that $D_{u, b}^{\mathrm{vert}}$ and $D_{u, b}$ have the same image.

Thus, the fiber $E_{b}$ of the obstruction bundle $E$ is coker $D_{u, b}^{\mathrm{vert}}$. The fundamental class of $\mathcal{M}_{0,0}^{*}\left(P_{f}, \sigma_{\max } ;\left\{J_{b}^{\text {reg }}\right\}\right)$ is identified with $\mathrm{PD}_{B \times F_{\max }} e(E)$, see [17, Chapter 7.2]. We thus have the following direct generalization of [19, Theorem 1.9].

Proposition 5.3 Let $f: B \rightarrow Q$ and the obstruction bundle $E$ be as above. Then

$$
\begin{aligned}
c^{q}\left(P_{f}\right)=\mathrm{PD}_{B \times F_{\max }} e(E) \otimes & q^{-m_{\max }} t^{H_{\max }} \\
& +\sum_{A \in H_{2}^{S}(M) \mid \omega(A)>0} b_{\sigma_{\max }+A} \otimes q^{-m_{\max }-c_{1}(A)} t^{H_{\max }-\omega(A)},
\end{aligned}
$$

where $m_{\max }=c_{\text {vert }}\left(\sigma_{x}\right)=\sum_{i} k_{i}$ and $H_{\max }$ is the maximum value of the normalized Hamiltonian generating $\gamma$.

Proof Since the evaluation map ev: $\mathcal{M}_{0,1}^{*}\left(P_{f}, \sigma_{\max },\left\{J_{b}\right\}\right) \rightarrow P_{f}$ intersects $I_{0}(B \times$ $M)$ transversally at $B \times F_{\max }$, it can be readily deduced from the above discussion that the class corresponding to the transverse intersection of

$$
\mathrm{ev}^{\mathrm{reg}}: \mathcal{M}_{0,1}^{*}\left(P_{f}, \sigma_{\mathrm{max}},\left\{J_{\text {reg }}\right\}\right) \rightarrow P_{f}
$$

with $I_{0}(B \times M)$ is $\mathrm{PD}_{B \times F} e(E) \in H_{*}(B \times M)$.

By [19, Lemma 3.1] there are no contributions from sections $\sigma_{\max }+A$ with $\omega(A)<0$; this also follows from the argument in the proof of Theorem 1.15.

To understand the obstruction bundle $E$, we need to understand cokernel of the linearized Cauchy-Riemann operator

$$
D_{u, b}^{\mathrm{vert}}: \Omega^{0}\left(S^{2}, u^{*} T X_{b}\right) \rightarrow \Omega^{0,1}\left(S^{2}, u^{*} T X_{b}\right),
$$

where $u: S^{2} \rightarrow X_{b}$ parametrizes the section $\sigma_{x} \in X_{b}, x \in F_{\max }$ as before.

The complex normal bundle $N\left(\sigma_{x}\right)$ of $\sigma_{x}$ inside $T X_{b}$ can be identified with the bundle

$$
\left(T_{x} M, J_{x}\right) \times_{S^{1}} S^{3} \rightarrow S^{2}
$$

and so splits into a sum of complex line bundles

$$
\bigoplus_{i=1}^{n} L_{k_{i}}
$$


where the degree of $L_{k_{i}}$ is $k_{i}$. In other words each $S^{1}$ invariant summand $V_{i} \simeq \mathbb{C}$ of $T_{x} M$, on which $S^{1}$ is acting by $v \mapsto e^{-2 \pi i k_{i} \theta} v$, gives rise to the summand $L_{k_{i}}$ of $N\left(\sigma_{x}\right)$. Thus,

$$
\left.T X_{b}\right|_{\sigma_{x}}=\left(\bigoplus_{i=1}^{n} L_{k_{i}}\right) \oplus L_{2} \equiv L,
$$

where $L_{2}$ is the tangent bundle to $\sigma_{x}$. Since $x \in F_{\max }, k_{i} \leq 0$ for all $z$.

By proof of [19, Lemma 3.2] the operator $D_{u_{x}, b}^{\text {vert }}$ is complex linear and is the Dolbeault operator $\bar{\partial}$ on $\left.T X_{\gamma}\right|_{\sigma_{x}}$, with respect to a holomorphic structure for which the splitting (27) is holomorphic. Thus, the cokernel of $D_{u_{x}, b}^{\text {vert }}$ is $H_{\bar{\partial}}^{0,1}\left(S^{2}, L\right) \simeq\left(H_{\bar{\partial}}^{1,0}\left(S^{2}, L^{*}\right)\right)^{*}$. The latter can be identified with $\left(H^{0}\left(S^{2}, L^{*} \otimes K_{x}\right)\right)^{*}$, where $K_{x}=T^{*}\left(\sigma_{x}\right)$ denotes the canonical bundle of $\sigma_{x}$.

Set

$$
E_{b, x, i}=H^{0}\left(S^{2}, L_{k_{i}}^{*} \otimes K_{x}\right) .
$$

This latter space can be identified with the space of degree $n_{i} \equiv-k_{i}-2$ homogeneous polynomials in $X, Y$, where $X, Y$ denote the homogeneous coordinates on $\mathbb{C} \mathbb{P}^{1}$. Thus, a section in $H^{0}\left(S^{2}, L_{k_{i}}^{*} \otimes K_{X}\right)$ is completely determined by its holomorphic $n_{i}$-jet over $0 \in D_{0}^{2} \subset S^{2}$. Therefore,

$$
E_{b, x, i} \simeq \bigoplus_{0 \leq j \leq n_{i}} \operatorname{Hom}\left(\left(T_{0} \sigma_{x}\right)^{\otimes j},\left.\left.K_{x}\right|_{0} \otimes L_{k_{i}}^{*}\right|_{0}\right) \simeq \bigoplus_{0 \leq j \leq n_{i}}\left(\left.K_{x}\right|_{0} ^{\otimes j}\right) \otimes\left(\left.\left.K_{x}\right|_{0} \otimes L_{k_{i}}^{*}\right|_{0}\right)
$$

The cokernel $E_{b, x}$ of $D_{u_{x}, b}^{\mathrm{vert}}$ is then

$$
E_{b, x}=\bigoplus_{i} E_{b, x, i}^{*}
$$

whose real dimension is the virtual index of $\gamma$, defined by

$$
I(\gamma)=\sum_{\substack{1 \leq i \leq n \\ k_{i} \leq-1}} 2\left(-k_{i}-1\right)
$$

Let $\widetilde{\mathcal{K}}$ be the bundle $Y \times_{S^{1}} \mathbb{C}$ and set $\mathcal{K}=\operatorname{pr}_{1}^{*} \widetilde{\mathcal{K}}$, where $\mathrm{pr}_{1}: B \times F_{\max } \rightarrow B$, and $\mathrm{pr}_{2}: B \times F_{\max } \rightarrow F_{\max }$ are the projections.

Then $\mathcal{K}$ is the bundle over $B \times F_{\max }$ whose fiber over $(x, b)=T_{0} \sigma_{x}=\left.K_{x}\right|_{0} ^{*}$, where $K_{x}=T^{*} \sigma_{x} \subset X_{b}$ (cf (14), (15)). We also have natural bundles $L_{i}$ over $B \times F_{\max }$ coming from the bundles $L_{k_{i}}$ above. 
Note that $e\left(L_{j}\right)$ and $e(\mathcal{K})$ are algebraically independent in the cohomology ring of $B \times M$. The Euler class of $E$ is given by

$$
\begin{aligned}
e(E) & =\prod_{i} \prod_{0 \leq j \leq n_{i}}\left((j+1) e(\mathcal{K})+e\left(L_{i}\right)\right) . \\
& =\prod_{i}\left(n_{i}+1\right) ! e^{\sum_{i}\left(n_{i}+1\right)}(\mathcal{K})+\text { mixed terms. }
\end{aligned}
$$

We can thus rewrite (28), using that $n_{i}=-k_{i}-2$, as

$$
e(E)=\sum_{0 \leq p \leq I(\gamma) / 2} e^{p}(\mathcal{K}) \cup a_{p},
$$

where $a_{p}$ are in $H^{I(\gamma) / 2-p}\left(B \times F_{\max }\right)$, consisting of sums of products of classes $e\left(L_{i}\right)$ with some coefficients.

Example 5.4 Let $\hat{f}: S^{3} \rightarrow \operatorname{Ham}\left(\mathbb{C} \mathbb{P}^{n}, \omega\right), \gamma: S^{1} \rightarrow \operatorname{Ham}\left(\mathbb{C} \mathbb{P}^{n}, \omega\right)$ and the associated map

$$
f_{h}: S^{2} \rightarrow Q=\left(L \operatorname{Ham}\left(\mathbb{C} \mathbb{P}^{n}, \omega\right) \times S^{\infty}\right) / S^{1}
$$

be as in Example 1.14. Then $F_{\max }=\max =[1,0, \ldots, 0], \mathcal{M}_{0,1}^{*}\left(P_{f_{h}}, \sigma_{\max } ;\left\{J_{b}\right\}\right)$ is identified with $S^{2}$ and the obstruction bundle $E$ is identified with the complex line bundle associated to the Hopf bundle $h: S^{3} \rightarrow S^{2}$, whose homological Euler class is $[-\mathrm{pt}] \in H_{0}\left(S^{2}\right)$. Thus, by Proposition 5.3

$$
c^{q}\left(P_{f_{h}}\right)=([-\mathrm{pt}] \otimes[\mathrm{pt}]) \otimes q^{-m_{\max }} t^{H_{\max }}+\text { lower } t \text {-order terms. }
$$

Theorem 5.5 Let $\widehat{f}: Y \rightarrow \operatorname{Ham}(M, \omega)$ be as above and $B=Y / S^{1}$. Every nonzero term

$$
e^{p}(\mathcal{K}) \cup a_{p} \in H^{*}\left(B \times F_{\max }\right)
$$

in the expansion for $e(E)$ gives rise to a nontrivial characteristic class $c_{2 p}^{q}\left(P_{f}\right)$. Moreover, it gives rise to cycles $f: C \rightarrow Q$, minimizing the positive max-length measure in their bordism class.

Proof If $e$ is the Euler class of $q: Y \rightarrow B$, then since $\mathcal{K}$ is isomorphic to $\operatorname{pr}_{1}^{*}\left(Y_{\gamma} \times S^{1}\right.$ $\mathbb{C})$ it follows that the Poincare dual of $e^{p}(\mathcal{K}) \cup a_{p}$ is of the form

$$
\mathrm{PD}_{B}(e) \otimes \mathrm{PD}_{F_{\text {max }}}\left(\left.a_{p}\right|_{[\mathrm{pt}] \times F_{\text {max }}}\right) \in H_{*}(B \times M),
$$

where $\operatorname{PD}(e) \in H_{*}(B)$ and $\left(\left.a_{p}\right|_{[\mathrm{pt}] \times F_{\max }}\right)^{*}$ is thought of as a class in $H_{*}(M)$ via inclusion of $F_{\max }$ into $M$. Since the generating function $H$ of $\gamma$ is necessarily a perfect Morse-Bott function (see McDuff and Salamon [16]) the inclusion of $F_{\max }$ into $M$ can be shown to be injective on homology. The first part of the theorem is then 
immediate from our assumption, the definition of the characteristic classes $c_{k}^{q}\left(P_{f}\right)$, and Proposition 5.3. We prove the second statement. For some $a \in H_{2 p}(B)$ we have that $0 \neq c_{2 p}^{q}\left(P_{f}\right)(a)$. By Proposition 4.1

$$
c_{2 p}^{q}\left(P_{f}\right)(a)=\Psi(f \circ g, C),
$$

where $g: C \rightarrow B$ is a smooth map representing the rational homology class of $a$. Thus, the cycle $f \circ g: C \rightarrow Q$ is essential in the bordism group by Lemma 3.8. Let us see that it minimizes the max-length measure. By Proposition 5.3, $v(\Psi(f \circ g, C))=H_{\max }$. On the other $L^{+}(f \circ g)=H_{\max }$, since all the loops in the image $\operatorname{Im}(f) \subset Q$ have positive Hofer length $H_{\max }$. By Proposition 5.1 $f \circ g$ minimizes the measure $L^{+}(f)$ in its bordism class.

\subsection{Proof of Theorem $\mathbf{1 . 1 0}$}

Since $e(\widetilde{\mathcal{K}})=e \neq 0$, the $p=I_{\gamma} / 2$ term in the expansion of $e(E)$ is nonzero. By Theorem 5.5, the cycle $f: B \rightarrow Q$ is essential and minimizes the measure $L^{+}(f)$ in its bordism class.

\subsection{Proof of Theorem 1.15}

Consider the fibration $h: S^{2 k+1} \rightarrow \mathbb{C P}^{k}$. Homotop $\hat{f}: S^{2 k+1} \rightarrow \operatorname{Ham}(M, \omega)$, so that it takes the set $h^{-1}\left(D^{c}\right)$ to id, where $D \subset \mathbb{C P}^{k}$ is an open ball. The new map will still be denoted by $\hat{f}$. Let $q: \mathbb{C P} \mathbb{P}^{k} \rightarrow S^{2 k}$ be the quotient map, squashing $\mathbb{C P}^{k}-D$ to $s_{0} \in S^{2 k}$. There is an induced quotient map

$$
\left.q\right|_{B} \times \text { id: }\left(h^{-1}(\bar{D}) \simeq \bar{D} \times S^{1}\right) \rightarrow S^{2 k} \times S^{1} .
$$

Since

$$
\widehat{f}\left((q \times \mathrm{id})^{-1}\left(s_{0} \times S^{1}\right)\right)=\widehat{f}\left(h^{-1}(\partial \bar{D})\right)=\mathrm{id},
$$

there is then an induced map

$$
\tilde{f}: S^{2 k} \times S^{1} \rightarrow \operatorname{Ham}(M, \omega)
$$

and the associated map

$$
f_{2}: S^{2 k} \rightarrow \text { LHam. }
$$

We will show now that $c^{q}\left(P_{f_{h}}\right)=c^{q}\left(P_{f_{2}}\right)$. On the other hand, we show in Lemma 5.7 below that $f$ is homotopy equivalent to $f_{2}$.

The restriction of $P_{f_{h}}$ to $D$ is the pullback by $q$ of the fibration $P_{f_{2}}$ over $S^{2 k}$. By (19), a section class $A \in H_{2}^{S}(X)$ contributes to $c^{q}\left(P_{f}\right)$ only if $c_{\text {vert }}(A) \leq 0$; moreover, if $c_{\text {vert }}(A)=0$, the class $A$ only contributes to the degree zero class $c_{0}^{q}(A)$ and so is not relevant to us. When $c_{\text {vert }}(A)<0$, the monotonicity of $M$ implies that $-\mathcal{C}(A)>0$ 
in this case, because $X \simeq M \times S^{2}$ (since $f$ and $f_{h}$ map into components of $Q$ corresponding to contractible loops in $\operatorname{Ham}(M, \omega)$ by construction). Put an admissible family $\left\{\Omega_{b}\right\}$ on $P_{f_{2}}$ as in Section 2.2, so that the area of the fiber $X_{b}$ over $b \in S^{2 k}$ is

$$
L^{+}(f(b))+2 \epsilon
$$

with $\epsilon<-\mathcal{C}(A)$. Let $\left\{J_{b}\right\}$ be a compatible regular family. The proof of Proposition 5.1 implies that the area of each fiber of $p: P_{f_{2}} \rightarrow S^{2 k}$ is at least $-\mathcal{C}(A)$ whenever there is a $\left\{J_{b}\right\}$-holomorphic $A$-curve in that fiber. Thus, no element of the moduli space $\mathcal{M}_{0}^{*}\left(P_{f_{2}}, A ;\left\{J_{b}\right\}\right)$ lies in the fiber over $s_{0}$, since the area of $\Omega_{s_{0}}$ is $2 \epsilon$.

Pullback by $q$ the families $\left\{\Omega_{b}\right\},\left\{J_{b}\right\}$ to $P_{f_{h}}$ over $\bar{D}$. The restriction of $\left\{q^{*} \Omega_{b}\right\}$ over $\partial \bar{D}$ is by construction the constant family restricting to a split symplectic form, ie $\omega+\pi^{*}(\alpha)$, with area $2 \epsilon$ on each fiber, since $\Omega_{s_{0}}$ has that property.

Since $\hat{f}$ is the constant map to id on $D^{c}$, the family $\left\{q^{*} \Omega_{b}\right\}$ over $\bar{D}$ can be extended to a family $\left\{\widetilde{\Omega}_{b}\right\}$ on $P_{f_{h}}$ such that the area of each fiber $X$ over $D^{c}$ is $2 \epsilon$. To see this note that the fibers of $\left.P_{f_{h}}\right|_{D_{c}}$ can be identified with the product $M \times S^{2}$, up to an action of $S^{1}$ which rotates the base $S^{2}$ and fixes $M$. Since the constant family $\left\{q^{*} \Omega_{b}\right\}$ over $\partial \bar{D}$ restricts to a split form on the fibers $X \simeq M \times S^{2}$, which is invariant under this $S^{1}$ action, there is an extension $\left\{\widetilde{\Omega}_{b}\right\}$ of $\left\{q^{*} \Omega_{b}\right\}$ to $D^{c}$. Pick any extension $\left\{\widetilde{J}_{b}\right\}$ of $\left\{q^{*}\left(J_{b}\right)\right\}$ which is compatible with $\left\{\widetilde{\Omega}_{b}\right\}$. By the above discussion, there are no $\left\{\widetilde{J}_{b}\right\}-$ holomorphic $A$-curves over $D^{c}$. Thus, $\left\{\widetilde{J}_{b}\right\}$ is regular, since it is regular for curves over $D$ as it is a pullback of a regular family $\left\{J_{b}\right\}$ there. Moreover, $q$ pushes forward the moduli space $\mathcal{M}_{0,1}^{*}\left(P_{f_{h}}, A ;\left\{\widetilde{J}_{b}\right\}\right)$ to the moduli space $\mathcal{M}_{0,1}^{*}\left(P_{f_{2}}, A,\left\{J_{b}\right\}\right)$ ie the diagram

$$
\mathcal{M}_{0,1}^{*}\left(P_{f_{h}}, A ;\left\{\widetilde{J}_{b}\right\} \stackrel{u \mapsto \widetilde{q} \circ u}{\longrightarrow} \mathcal{M}_{0,1}^{*}\left(P_{f_{2}}, A ;\left\{J_{b}\right\}\right)\right.
$$

commutes, where $\tilde{q}$ is a lift of $q$ which is defined on $\left.P_{f_{h}}\right|_{D}$.

By definition,

$$
\begin{gathered}
c^{q}\left(P_{f_{2}}\right)=\sum_{A} b_{A} \otimes e^{A} \in Q H_{*}^{S^{2 k}}(M), \\
c^{q}\left(P_{f_{h}}\right)=\sum_{A} b_{A}^{\prime} \otimes e^{A} \in Q H_{*}^{\mathbb{C} \mathbb{P}^{k}}(M),
\end{gathered}
$$

where $b_{A}$ is the transverse intersection of

$$
\text { ev: } \mathcal{M}_{0,1}^{*}\left(P_{f_{h}}, A,\left\{\tilde{J}_{b}\right\}\right) \rightarrow P_{f_{h}}
$$


with $I_{0}\left(\mathbb{C} \mathbb{P}^{k} \times M\right)$, and $b_{A}^{\prime}$ is the transverse intersection of

$$
\text { ev: } \mathcal{M}_{0,1}^{*}\left(P_{f_{2}}, A,\left\{J_{b}\right\}\right) \rightarrow P_{f_{2}}
$$

with $I_{0}\left(S^{2 k} \times M\right)$. Since the above moduli spaces lie over contractible subsets of $\mathbb{C P}^{k}$ and $S^{2 k}$

$$
\begin{array}{r}
b_{A}=[\mathrm{pt}] \otimes b_{A}^{M} \in H_{*}\left(S^{2 k} \times M\right) \\
b_{A}^{\prime M}=[\mathrm{pt}] \otimes b_{A}^{\prime M} \in H_{*}\left(\mathbb{C P}^{k} \times M\right),
\end{array}
$$

for some $b_{A}, b_{A}^{\prime} \in H_{*}(M)$. The above discussion implies that $b_{A}^{M}=b_{A}^{\prime M}$. Thus the two total classes are the same. To conclude that the only nonvanishing classes of the two fibrations are in the top dimension note that $[\mathrm{pt}] \otimes b_{A}^{M}$ has a nontrivial intersection pairing with $c \otimes b \in H_{*}\left(S^{2 k} \times M\right)$ only if $c=\left[S^{2 k}\right] \in H_{*}\left(S^{2 k}\right)$ and use definition of the classes.

Remark 5.6 This proof makes extensive use of monotonicity. It is not obvious to me if this theorem is true in a situation where one must use methods of the virtual moduli cycle.

Lemma 5.7 The maps $f$ and $f_{2}$ above are freely homotopy equivalent.

Proof The map $f$ is induced from a composition of maps of pairs

$$
\left(D^{2 k} \times S^{1}, \partial D^{2 k} \times S^{1}\right) \stackrel{t}{\rightarrow}\left(S^{2 k+1}, \mathrm{pt}\right) \stackrel{\widehat{f}}{\rightarrow}(\operatorname{Ham}(M, \omega), \mathrm{id}) .
$$

On the other hand, $f_{2}$ is the induced map from the composition of maps of pairs

$$
\left(D^{2 k} \times S^{1}, \partial D^{2 k} \times S^{1}\right) \stackrel{i}{\rightarrow}\left(S^{2 k+1}, h^{-1}\left(D^{c}\right)\right) \stackrel{\widehat{f}}{\rightarrow}(\operatorname{Ham}(M, \omega), \mathrm{id}) .
$$

Clearly, we can homotop $\hat{f}$ through maps of pairs to a map $\hat{f}^{\prime}:\left(S^{2 k+1}, O^{c}\right) \rightarrow$ $\left(\operatorname{Ham}(M, \omega)\right.$, id), where $O \subset h^{-1}(D)$ is an open ball which does not contain [pt]. Then $f$ is homotopic to a map induced from the composition

$$
\left(D^{2 k} \times S^{1}, \partial D^{2 k} \times S^{1}\right) \stackrel{i}{\rightarrow}\left(S^{2 k+1}, O^{c}\right) \stackrel{\hat{f}^{\prime}}{\rightarrow}(\operatorname{Ham}(M, \omega), \mathrm{id}),
$$

and $f_{2}$ is induced from

$$
\left(D^{2 k} \times S^{1}, \partial D^{2 k} \times S^{1}\right) \stackrel{t}{\rightarrow}\left(S^{2 k+1}, O^{c}\right) \stackrel{\hat{f}^{\prime}}{\rightarrow}(\operatorname{Ham}(M, \omega), \mathrm{id}) .
$$

Thus, we just need to show that $i$ is homotopic via maps of pairs to $t$. To see this one can use degree. 


\section{The Hopf algebra structure of $H_{*}(L$ Ham, $\mathbb{Q})$}

This section is mostly an excursion, which studies the relationship of the homomorphism $\Psi$ with the Pontryagin ring structure of $H_{*}(L H a m, \mathbb{Q})$. It may be interesting to the reader in order to get an idea of how the use of $S^{1}$-symmetry in the previous section relates to the bigger picture of QC classes.

The Milnor-Moore theorem states that a connected co-commutative Hopf algebra $A$ over a field of characteristic zero is generated by its primitive elements. A primitive element is an element $a \in A$ such that its coproduct is $1 \otimes a+a \otimes 1$. More precisely it says that $A$ is isomorphic as a Hopf algebra to the universal enveloping algebra $\mathcal{U}(P(A))$, where $P(A)$ denotes the associated Lie algebra of its primitive elements. In other words the only relations in $\mathcal{U}(P(A))$ are the ones of the form

$$
a \otimes b-(-1)^{p q} b \otimes a=a b-(-1)^{p q} b a,
$$

where the product on the right is the product in the Hopf algebra. When $A$ is the rational Hopf algebra of an $H$-space, Cartan-Serre theorem states that the Lie algebra of primitive elements consists of spherical classes. In fact, we have the following.

Theorem 6.1 (Milnor-Moore [20], Cartan-Serre [3]) Let $X$ be a connected $H-$ space. Denote by $\pi_{*}(X, \mathbb{Q}) \subset H_{*}(X, \mathbb{Q})$ the Lie subalgebra of the associated algebra of the ring, generated by the image of the Hurewitz map $h: \pi_{*}(X) \rightarrow H_{*}(X, \mathbb{Q})$ and denote by $\mathcal{U}\left(\pi_{*}(X, \mathbb{Q})\right)$ the universal enveloping algebra of $\pi_{*}(X, \mathbb{Q})$. Then

$$
H_{*}(X, \mathbb{Q}) \simeq \mathcal{U}\left(\pi_{*}(X, \mathbb{Q})\right),
$$

as rings (in fact as Hopf algebras).

For $[\gamma] \in \pi_{1}\left(\operatorname{Ham}(M, \omega)\right.$, id), let $L^{[\gamma]} \subset L$ Ham denote the component containing the loop $\gamma$. As a space

$$
L^{[\gamma]}=\Omega^{[\gamma]} \operatorname{Ham}(M, \omega) \times \operatorname{Ham}(M, \omega),
$$

where $\Omega^{[\gamma]} \operatorname{Ham}(M, \omega)$ denotes the $\gamma$-component of the based loop space at id. Hence,

$$
\pi_{*}\left(X^{[\gamma]}\right) \simeq \pi_{*}(\operatorname{Ham}(M, \omega)) \oplus \pi_{*}\left(\Omega^{[\gamma]} \operatorname{Ham}(M, \omega)\right) .
$$

Combining this with Theorem $6.1\left(L^{[\gamma]}\right.$ is not a connected $H$-space naturally but is homeomorphic to one), we get

$$
\left.H_{*}\left(L^{[\gamma]}, \mathbb{Q}\right) \simeq \mathcal{U}\left(\pi_{*}(\operatorname{Ham}(M, \omega), \mathbb{Q})\right) \otimes \mathcal{U}\left(\pi_{*}\left(\Omega^{[\gamma]} \operatorname{Ham}(M, \omega)\right), \mathbb{Q}\right)\right)
$$


as rings. By Lemma 6.2 below, $\Psi$ vanishes on

$$
H_{*}(\operatorname{Ham}(M, \omega), \mathbb{Q}) \simeq \mathcal{U}\left(\pi_{*}(\operatorname{Ham}(M, \omega), \mathbb{Q})\right)
$$

for $*>0$. On the other hand $\Psi\left(H_{0}(\operatorname{Ham}(M, \omega), \mathbb{Q})\right.$ is generated over $\mathbb{Q}$ by $[M]$, the multiplicative identity element; see Remark 4.3.

If one is to extend $\Psi$ to a map

$$
\Psi: H_{*}(L \operatorname{Ham}, \mathbb{Q}) \rightarrow Q H_{*+2 n}(M),
$$

which can likely be done using pseudocycles, the above discussion together with Theorem 1.9 shows that $\Psi$ would only be interesting on

$$
H_{*}(\Omega \operatorname{Ham}(M, \omega), \mathbb{Q})=\bigoplus_{\gamma} \mathcal{U}\left(\pi_{*}\left(\Omega^{[\gamma]} \operatorname{Ham}(M, \omega)\right) \subset H_{*}(L \operatorname{Ham}, \mathbb{Q}),\right.
$$

a direct sum over $[\gamma]$ of free graded commutative algebras on $\pi_{*}(\Omega \operatorname{Ham}(M, \omega))$. At the same time, working on the free loop space allows us to pass to the $S^{1}$ equivariant setting, using which we were able to do computations in Section 5.

Define $i^{[\gamma]}: \operatorname{Ham}(M, \omega) \rightarrow L^{\gamma}$ to be the inclusion which takes an element $\phi \in$ $\operatorname{Ham}(M, \omega)$ to the loop $\phi \circ \gamma$.

Lemma 6.2 If $k>0, \Psi(f)=0$ for $f: B^{k} \rightarrow i^{[\gamma]}(\operatorname{Ham}(M, \omega))$, where .

Proof This follows from the fact that for a map

$$
\begin{aligned}
f: B^{k} & \rightarrow i_{\gamma}(\operatorname{Ham}(M, \omega)) \subset L^{\gamma} \\
f(b) & =\phi_{b} \circ \gamma, \quad \text { where } \phi_{b} \in \operatorname{Ham}(M, \omega),
\end{aligned}
$$

the fibration $P_{f}$ is isomorphic to a trivial $\mathcal{F}$-fibration by an isomorphism which is a Hamiltonian bundle map on each fiber, and so the relevant invariants vanish by Axiom 1 . Let $c_{[\gamma]}$ be the constant map $f: B \rightarrow L^{[\gamma]}$ to the loop $\gamma$. We trivialize $P_{f}$ as follows:

$$
\begin{aligned}
& P_{f}=\left(B \times M \times D^{2}\right)_{0} \cup_{f}\left(B \times M \times D^{2}\right)_{\infty} \\
& \stackrel{\operatorname{tr}}{\rightarrow} P_{c_{[\gamma]}}=\left(B \times M \times D^{2}\right)_{0}^{\prime} \cup_{c_{[\gamma]}}\left(B \times M \times D^{2}\right)_{\infty}^{\prime}, \\
& \operatorname{tr}(b, x, z)_{0}:=(b, x, z)_{0}^{\prime} \quad \text { and } \operatorname{tr}(b, x, z)_{\infty}:=\left(b, \phi_{b}^{-1}(x), z\right)_{\infty}^{\prime},
\end{aligned}
$$

where $\phi_{b} \in \operatorname{Ham}(M, \omega)$ is as above. This map is easily seen to be well defined. 


\section{Structure group of $\mathcal{F}$-fibrations}

This section is concerned with the structure groups of the fibrations $\tilde{p}$ : $U \rightarrow L \mathrm{Ham}$ and $p: U^{S^{1}} \rightarrow Q$, which is indirectly used for the proof of Lemma 2.5. Another, perhaps more pertinent goal here is to prove that $\tilde{p}: U \rightarrow L$ Ham is universal for its structure group.

Proposition 7.1 The structure group of $p: U \rightarrow L$ Ham over the component of the loop $\gamma$ can be reduced to the group $\mathcal{F}^{\gamma}$ of Hamiltonian bundle maps of $X_{\gamma}$ which are identity over $D_{0}^{2}$ and over a small neighborhood of $0 \in D_{\infty}^{2}$ in coordinates of (3).

This proposition follows immediately from Lemma 7.4 proved later in this section. For $\gamma: S^{1} \rightarrow \operatorname{Ham}(M, \omega)$ let $[\gamma]$ denote its equivalence class in $\pi_{1}(\operatorname{Ham}(M, \omega)$,id).

Proposition 7.2 Let $Q^{[\gamma]}$ denote a connected component of $Q$. The structure group of $p: U^{[\gamma]} \rightarrow Q^{[\gamma]}$ may be reduced to the group of Hamiltonian bundle maps of $\pi_{\gamma}: X_{\gamma} \rightarrow$ $S^{2}$, which sit over rotations the base $S^{2}$, with the axis of rotation corresponding to $0 \in D_{0}^{2}, 0 \in D_{\infty}^{2}$. Moreover, elements of this group act as id $\times$ rot on $M \times D_{0}^{2} \subset X_{\gamma}$ and by identity on the fiber over $0 \in D_{\infty}^{2}$.

The proof will be given after some preliminaries. To make the discussion more transparent we work with connections, which to us will be just smooth or continuous functors. In fact, there is a natural such connection on $\tilde{p}: U \rightarrow L$ Ham.

\subsection{The path groupoid}

A topological category is a small category in which the set of all objects and the set of all morphisms are topologized, so that the source and target maps and all structure maps are continuous. Let $p: P \rightarrow B$ be a bundle with fiber $X$, where $B$ is a topological group. Let $\mathcal{C}(B)$ be a topological groupoid whose objects are the points of $B$. The morphisms from $a$ to $b$ are defined to be

$$
\mathcal{C}(a, b)=P(a, b),
$$

the space of continuous paths from $a$ to $b$, ie maps $m:[0,1] \rightarrow B$ s.t. $m(0)=a$ and $m(1)=b$. The composition law

$$
\mathcal{C}(a, b) \times \mathcal{C}(b, c) \rightarrow \mathcal{C}(a, c)
$$

is defined as follows. Let $m_{a, b}:[0,1] \rightarrow B$ be a path with endpoints $a, b$ and $m_{b, c}:[0,1] \rightarrow B$ be a path with endpoints $b, c$. Then $m_{b, c} \circ m_{a, b}:[0,1] \rightarrow B$ is defined by

$$
m_{b, c} \circ m_{a, b}(t)=m_{b, c}(t) \cdot\left(m_{b, c}(0)\right)^{-1} \cdot m_{a, b}(t) .
$$


This path clearly has endpoints $a, c$ and is continuous. This is essentially the only natural way to define a composition law for paths in a topological group. The topology on the set of morphisms ie the free path space of $B$ is taken to be the compact open topology.

\subsection{The category $\mathcal{D}(P, B, p)$}

We also define a topological category $\mathcal{D}(P, B, p)$, whose space of objects is homeomorphic to $B$ with elements: manifolds $X_{b}=p^{-1}(b)$ for $b \in B$. The space of morphisms from $X_{a}$ to $X_{b}$ is defined to be

$$
\mathcal{D}\left(X_{a}, X_{b}\right)=\operatorname{Homeo}\left(X_{a}, X_{b}\right),
$$

the space of homeomorphisms from $X_{a}$ to $X_{b}$. The composition law is just the composition of homeomorphisms.

7.2.1 Topology on the space of morphisms of $\mathcal{D}(P, B, p)$ For each $b \in B$, let $U_{b} \subset B$ be an open set with a trivialization $\phi_{b}: U_{b} \times X \rightarrow p^{-1}\left(U_{b}\right)$. Let now $a, b \in B$. Any morphism whose source is the fiber $X_{u_{1}}$ with $u_{1} \in U_{a}$ and target $X_{u_{2}}$ with $u_{2} \in U_{b}$ can be identified via the trivializations $\phi_{a}, \phi_{b}$ with a homeomorphism from $X$ to $X$. Thus, the set of such morphisms is identified with $U_{a} \times U_{b} \times \operatorname{Homeo}(X, X)$, which we will denote by $\mathcal{D}\left(U_{a}, U_{b}\right)$. It has a natural topology, where the topology on $\operatorname{Homeo}(X, X)$ is the compact-open topology. The basis for a topology on the set of all morphism then consists of open sets in $\mathcal{D}\left(U_{a}, U_{b}\right)$ for all $a, b \in B$. Clearly, a different choice of trivializations gives rise to equivalent topologies.

\subsection{Connections}

Definition 7.3 Let $p: P \rightarrow B$ be as above. An abstract connection is defined to be a continuous functor $F$ from the category $\mathcal{C}(B)$ to $\mathcal{D}(P, B, p)$.

The map $F(m): p^{-1}\left(m_{0}\right) \rightarrow p^{-1}\left(m_{1}\right)$ will be called the parallel transport map. The name of the connection is the name of the corresponding functor (eg $F$ ). The word abstract in abstract connection will often be dropped. We may define the holonomy group of an abstract connection exactly the same way as for usual smooth connections on $G$-bundles, using the parallel transport maps.

Lemma 7.4 The structure group of $p: P \rightarrow B$ over a connected component can be reduced to the holonomy group $\operatorname{Hol}(F)$ of the connection $F$ on this component. 
Proof Let $\left\{U_{i}\right\}$ be a cover of $B$ by contractible open sets and $H_{i}: U_{i} \times I \rightarrow B$ be a free homotopy, which at time 0 is the constant map to $b_{0}$ and at time 1 is the inclusion map of $U_{i}$. Then parallel translating by $F$, along the paths of the homotopy $h_{i, x}(t)=H_{i}(x, t)$, gives a trivialization $\operatorname{tr}_{i}: U_{i} \times X \rightarrow p^{-1}\left(U_{i}\right)$. The transition map $\operatorname{tr}_{i j}: U_{i} \bigcap U_{j} \times X \rightarrow U_{i} \bigcap U_{j} \times X$ is by construction and functoriality of $F$ given by parallel translation by $F$ along the loops $h_{j, x}^{-1} \circ h_{i, x}$. Here $\circ$ is the multiplication in the groupoid $\mathcal{C}(B)$.

\subsection{A connection $F_{U}$ on $p: U \rightarrow L$ Ham}

The space $L$ Ham is a topological group and we may take the topological groupoid $\mathcal{C}($ LHam $)$ defined as above, except that we take the morphisms in the groupoid to be smooth in the sense below.

Definition 7.5 We define a map $m:[0,1] \rightarrow L$ Ham to be smooth if it is locally constant at the endpoints and the associated map $\tilde{m}:[0,1] \times S^{1} \rightarrow \operatorname{Ham}(M, \omega)$ is smooth.

The groupoid $\mathcal{C}(L \mathrm{Ham})$ is topologized as a subspace of continuous maps with its compact open topology.

The parallel transport map Let $m: I \rightarrow L$ Ham be a path. We define the map $F_{U}(m)=t_{m}$ from the fiber $X_{m_{0}}$ over $m(0)=m_{0}$, to the fiber $X_{m_{1}}$ over $m(1)=m_{1}$ as follows. We have

$$
\begin{aligned}
& X_{m_{0}}=M \times D_{0}^{2} \cup_{m_{0}} M \times D_{\infty}^{2}, \\
& X_{m_{1}}=M \times D_{0}^{2} \cup_{m_{1}} M \times D_{\infty}^{2}
\end{aligned}
$$

If $r, \theta$ are polar coordinates on $D^{2}$, then

$$
t_{m}(x, r, \theta)_{0}=(x, r, \theta)_{0} \quad \text { and } \quad t_{m}(x, r, \theta)_{\infty}=\left(m_{r, \theta} \circ m_{0, \theta}^{-1}(x), r, \theta\right),
$$

where $m_{r, \theta}$ denotes the element of the loop $m_{r}=m(r)$ at time $\theta$.

This is well defined under the gluing since the diagram

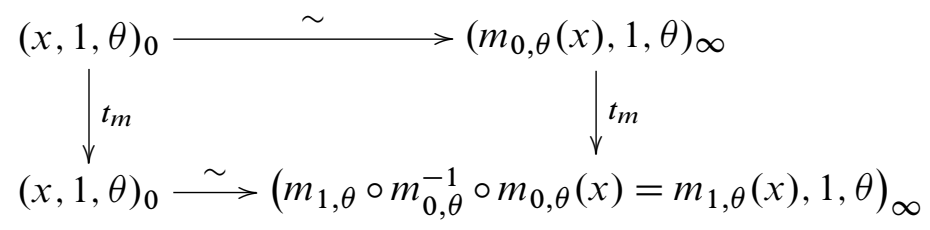

commutes. We leave it to the reader to verify that this gives a continuous functor $F_{U}: \mathcal{C}(L$ Ham $) \rightarrow \mathcal{D}(U, L$ Ham, $p)$, which assigns to $\gamma \in L$ Ham the fiber $X_{\gamma}$ and to 
a morphism $m: I \rightarrow L$ Ham from $\gamma_{0}$ to $\gamma_{1}$ the map $t_{m}: X_{\gamma_{0}} \rightarrow X_{\gamma_{1}}$. We denote by $L \mathrm{Ham}^{\gamma}$ the component of the loop $\gamma$ in LHam.

Lemma 7.6 The group $\operatorname{Hol}\left(F_{U}\right)$ is isomorphic to the group $\mathcal{C}(\gamma, \gamma)$ of automorphisms of the object $\gamma$ in $\mathcal{C}(L \mathrm{Ham})$.

Proof By construction of the connection $F_{U}$, the natural surjective holonomy map hol: $\operatorname{Aut}_{\mathcal{C}}(\gamma) \rightarrow \operatorname{Hol}(F)$ has no kernel.

Let $E$ denote the space of all smooth paths in LHam based at $\gamma$ (see Definition 7.5). This is a contractible space with a free continuous action of the group $\operatorname{Aut}_{\mathcal{C}}(\gamma)$ acting by left multiplication using the topological groupoid structure of $\mathcal{C}(L \mathrm{Ham})$. Moreover, this action fixes the fibers of the projection $k: E \rightarrow L$ Ham given by evaluating at the endpoint and is transitive on the fibers. It follows that $k: E \rightarrow L$ Ham is the universal $\operatorname{Aut}_{\mathcal{C}}(\gamma)$-bundle. In other words

$$
B \operatorname{Aut}_{C}(\gamma)=B \operatorname{Hol}\left(F_{U}\right)=L \operatorname{Ham}^{\gamma} .
$$

In fact, we have the following:

Proposition 7.7 The bundle $\tilde{p}: U \rightarrow L \mathrm{Ham}^{\gamma}$ is the associated bundle to the universal principal $\operatorname{Aut}_{\mathcal{C}}(\gamma)$-bundle $k: E \rightarrow L \mathrm{Ham}^{\gamma}$.

Proof This follows from the proof of Lemma 7.4. The details are left to the reader. $\square$

\subsection{Proof of Proposition 7.2}

Recall that the bundle $p: U^{S^{1}} \rightarrow Q$ is the quotient by the $S^{1}$ action $\tilde{\rho}$ of the bundle $p \times$ id: $U \times S^{\infty} \rightarrow L$ Ham $\times S^{\infty}$ (cf Section 2.1). Let $V_{i}$ be a contractible open set in $Q^{\gamma}$ and

$$
g_{i}: V_{i} \times S^{1} \rightarrow L \operatorname{Ham} \times S^{\infty}
$$

a local trivialization of the principal $S^{1}$ bundle $h: L \mathrm{Ham} \times S^{\infty} \rightarrow Q^{\gamma}$. Let $H_{i}$ be a free homotopy of the map $g_{i}: V_{i} \times 0 \rightarrow L \mathrm{Ham} \times S^{\infty}$ to the constant map to $\left(\gamma_{0}, s_{0}\right)$. As before, the connection $F$ then induces a map

$$
t_{i}: V_{i} \times X_{\gamma_{0}} \rightarrow p^{-1}\left(g_{i}\left(U_{i} \times 0\right)\right),
$$

by parallel translating along the paths of the homotopy $H_{i}$. The transition maps $t_{i j}$ have the form:

$$
t_{i j}(u, x)=\left(u, t_{j}^{-1} \circ \tilde{\rho}\left(\theta_{i j}\right)^{-1} \circ t_{i}(x)\right)
$$


where $\theta_{i j}$ comes from the transition maps $g_{i j}: V_{i} \cap V_{j} \times S^{1} \rightarrow V_{i} \cap V_{j} \times S^{1}, g_{i j}(u, \theta)=$ $\left(u, \theta+\theta_{i j}\right)$. By construction, this is a Hamiltonian bundle map of $\pi_{\gamma}: X_{\gamma} \rightarrow S^{2}$ to itself which sits over the rotation by $\theta_{i j}$ of the base and fixes the fibers over 0 and infinity.

\section{References}

[1] J C Álvarez Paiva, A C Thompson, Volumes on normed and Finsler spaces, from: "A sampler of Riemann-Finsler geometry", Math. Sci. Res. Inst. Publ. 50, Cambridge Univ. Press (2004) 1-48 MR2132656

[2] O Buse, Relative family Gromov-Witten invariants and symplectomorphisms arXiv: $\mathrm{SG} / 01110313$

[3] H Cartan, J-P Serre, Espaces fibrés et groupes d'homotopie II: Applications, C. R. Acad. Sci. Paris 234 (1952) 393-395 MR0046046

[4] H Federer, Geometric measure theory, Die Grund. der math. Wissenschaften 153, Springer, New York (1969) MR0257325

[5] H Hofer, On the topological properties of symplectic maps, Proc. Roy. Soc. Edinburgh Sect. A 115 (1990) 25-38 MR1059642

[6] M Hutchings, Floer Homology of families I arXiv:math/0308115

[7] M Hutchings, Floer Homology of families II, in preparation

[8] E-N Ionel, T H Parker, The symplectic sum formula for Gromov-Witten invariants, Ann. of Math. (2) 159 (2004) 935-1025 MR2113018

[9] J Kędra, D McDuff, Homotopy properties of Hamiltonian group actions, Geom. Topol. 9 (2005) 121-162 MR2115670

[10] F Lalonde, D McDuff, The geometry of symplectic energy, Ann. of Math. (2) 141 (1995) 349-371 MR1324138

[11] F Lalonde, D McDuff, Hofer's $L^{\infty}$-geometry: energy and stability of Hamiltonian flows I, II, Invent. Math. 122 (1995) 1-33, 35-69 MR1354953

[12] A-M Li, Y Ruan, Symplectic surgery and Gromov-Witten invariants of Calabi-Yau 3-folds, Invent. Math. 145 (2001) 151-218 MR1839289

[13] D McDuff, Private communication

[14] D McDuff, Quantum homology of fibrations over $S^{2}$, Internat. J. Math. 11 (2000) 665-721 MR1780735

[15] D McDuff, Geometric variants of the Hofer norm, J. Symplectic Geom. 1 (2002) 197-252 MR1959582 
[16] D McDuff, D Salamon, Introduction to symplectic topology, second edition, Oxford Math. Monographs, The Clarendon Oxford University Press, New York (1998) MR1698616

[17] D McDuff, D Salamon, J-holomorphic curves and symplectic topology, American Math. Society Colloquium Publ. 52, Amer. Math. Soc. (2004) MR2045629

[18] D McDuff, J Slimowitz, Hofer-Zehnder capacity and length minimizing Hamiltonian paths, Geom. Topol. 5 (2001) 799-830 MR1871405

[19] D McDuff, S Tolman, Topological properties of Hamiltonian circle actions, IMRP Int. Math. Res. Pap. (2006) 1-77 MR2210662

[20] J W Milnor, J C Moore, On the structure of Hopf algebras, Ann. of Math. (2) 81 (1965) 211-264 MR0174052

[21] J W Milnor, J D Stasheff, Characteristic classes, Annals of Math. Studies 76, Princeton University Press (1974) MR0440554

[22] A G Reznikov, Characteristic classes in symplectic topology, Selecta Math. (N.S.) 3 (1997) 601-642 MR1613528 Appendix D by L Katzarkov

[23] Y Savelyev, Virtual Morse theory on $\Omega \operatorname{Ham}(M, \omega)$, in progress

[24] P Seidel, $\pi_{1}$ of symplectic automorphism groups and invertibles in quantum homology rings, Geom. Funct. Anal. 7 (1997) 1046-1095 MR1487754

[25] I Ustilovsky, Conjugate points on geodesics of Hofer's metric, Differential Geom. Appl. 6 (1996) 327-342 MR1422339

[26] C Vafa, Topological mirrors and quantum rings, from: "Essays on mirror manifolds", Int. Press, Hong Kong (1992) 96-119 MR1191421

Stony Brook University

Department of Mathematics, Stony Brook, NY 11790, USA

yasha@math.sunysb.edu

http://www. math.sunysb.edu/ yasha

Proposed: Leonid Polterovich

Seconded: Yasha Eliashberg, Simon Donaldson
Received: 9 February 2008

Revised: 18 July 2008 